\title{
KIF21A-Mediated Axonal Transport and Selective Endocytosis Underlie the Polarized Targeting of NCKX2
}

\author{
Kyu-Hee Lee, ${ }^{1}$ Jae Sung Lee, ${ }^{1}$ Doyun Lee, ${ }^{1}$ Dae-Hyun Seog, ${ }^{2}$ Jonathan Lytton, ${ }^{3}$ Won-Kyung Ho, ${ }^{1}$ and Suk-Ho Lee \\ ${ }^{1}$ Department of Physiology and bioMembrane Plasticity Research Center, Seoul National University College of Medicine, Seoul 110-799, South Korea, \\ ${ }^{2}$ Department of Biochemistry, College of Medicine, Inje University, Busan 633-165, South Korea, and 3Department of Biochemistry and Molecular Biology, \\ Libin Cardiovascular Institute of Alberta, Hotchkiss Brain Institute, University of Calgary, Calgary, Alberta T2N 4N1, Canada
}

We have previously shown that $\mathrm{K}^{+}$-dependent $\mathrm{Na}^{+} / \mathrm{Ca}^{2+}$ exchanger (NCKX) is a major calcium clearance mechanism at the large axon terminals of central neurons, whereas their somata display little NCKX activity. We investigated mechanisms underlying the axonal polarization of NCKX2 in rat hippocampal neurons. We identified NCKX2 as the first neuron-specific cargo molecule of kinesin family member 21A (KIF21A). The intracellular loop of NCKX2 specifically interacted with the WD-40 repeats, a putative cargo-binding domain, of KIF21A. Dominant-negative mutant or depletion of KIF21A inhibited the transport of NCKX2-GFP to axon fibers. Knockdown of KIF21A caused calcium dysregulation at axonal boutons but not at somatodendritic regions. Despite the axonal polarization of the NCKX activity, both somatodendritic and axonal regions were immunoreactive to NCKX2. The surface expression of NCKX2 revealed by live-cell immunocytochemistry, however, displayed highly polarized distribution to the axon. Inhibition of endocytosis increased the somatodendritic surface NCKX2 and thus abolished the axonal polarization of surface NCKX2. These results indicate that KIF21A-mediated axonal transport and selective somatodendritic endocytosis underlie the axonal polarized surface expression of NCKX2.

\section{Introduction}

Calcium plays the most versatile signaling messenger in a neuron. Together with calcium influx and buffers, calcium clearance mechanisms are essential factors determining cytosolic calcium dynamics. There is accumulating evidence for crucial roles of calcium clearance mechanisms in synaptic plasticity and $\mathrm{Ca}^{2+}$ homeostasis (Jeon et al., 2003; Li et al., 2006; D. Lee et al., 2007). The somatodendritic and axonal regions in a neuron display distinct spectra of membrane proteins that serve specialized functions in each compartment. Although there are many studies about polarized expression of ion channels and receptors for neurotransmitters (Arnold, 2007; Hirokawa et al., 2010), little is known about polarization of calcium clearance mechanisms. Previously, we have reported that $\mathrm{K}^{+}$-dependent $\mathrm{Na}^{+} / \mathrm{Ca}^{2+} \mathrm{ex}$ changer (NCKX) is the most important calcium clearance mechanism at the axon terminals of central neurons, whereas the $\mathrm{Na}^{+} /$ $\mathrm{Ca}^{2+}$ exchanger $(\mathrm{NaCaX})$ activity in the somata was independent of intracellular K ${ }^{+}$(S.-H. Lee et al., 2002, 2007, 2009; Kim et al., 2003, 2005). Despite evidence for the role of NCKX in large axon terminals, it is still obscure whether NCKX plays a role in calcium

\footnotetext{
Received Dec. 20, 2011; revised Jan. 18, 2012; accepted Feb. 1, 2012

Author contributions: K.-H.L. and S.-H.L. designed research; K.-H.L., J.S.L., D.L., J.L., and S.-H.L. performed research; D.-H.S. contributed unpublished reagents/analytic tools; K.-H.L., J.S.L., J.L., W.-K.H., and S.-H.L. analyzed data; K.-H.L., W.-K.H., and S.-H.L. wrote the paper.

This work was supported by Korea Institute of Science and Technology Research and Development institutional program, Brain Research Center Grant 2009K001247, and bioMembrane Plasticity Research Center Grant 0633-2010-0003.

The authors declare no competing financial interests.

Correspondence should be addressed to Dr. Suk-Ho Lee, Department of Physiology, Seoul National University College of Medicine, 103 Daehak-ro, Jongno-gu, Seoul 110-799, South Korea. E-mail: leesukho@snu.ac.kr.

DOI:10.1523/JNEUROSCI.6331-11.2012

Copyright $\odot 2012$ the authors $\quad 0270-6474 / 12 / 324102-16 \$ 15.00 / 0$
}

clearance at a small axon terminal. Furthermore, molecular mechanisms underlying axonal restriction of NCKX activity has not been investigated. Among members of the NCKX family, only NCKX2 is brain specific (Tsoi et al., 1998), and the knockout of NCKX2 has profound effects on motor learning and ischemic brain damage (Li et al., 2006; Cuomo et al., 2008). Several lines of evidence support the notion that NCKX2 is a major isoform responsible for the NCKX activity at axon terminals (see Discussion). Therefore, we investigated mechanisms underlying polarized action of NCKX2 in hippocampal neurons.

There are two prevailing hypotheses for the mechanism underlying polarization of axonal proteins: (1) axonal transport by a motor protein that differentiates axonal microtubules from somatodendritic ones (selective trafficking) (Hirokawa and Takemura, 2005); (2) preferential endocytosis from the somatodendritic surface (selective retention) (Sampo et al., 2003). In any case, selective entry of a kinesin superfamily (KIF)-cargo complex into the axonal compartment through the axon initial segment (AIS) filter seems to be essential for axonal targeting (Song et al., 2009). We identified KIF21A as a putative binding partner of the intracellular loop of NCKX2. We present here the compelling evidence that KIF21A is essential for the axonal transport of NCKX2. The expression level of KIF21A is high in axon-rich tissues similar to KIF3A and nKHC, molecules known to participate in axonal transport (Marszalek et al., 1999; Hirokawa et al., 2010; Silverman et al., 2010). Point mutations in KIF21A were found in patients of congenital cranial dysinnervation disorders (Yamada et al., 2003; Heidary et al., 2008). Although the genetic disorder implies that KIF21A is involved in axonal transport, no neuronspecific cargo of KIF21A has been identified. We report here 
NCKX2 as the first neuron-specific cargo molecule transported to the axon by KIF21A. Furthermore, we present evidence supporting the selective retention hypothesis as the mechanism for polarized axonal surface expression of NCKX2.

\section{Materials and Methods}

DNA constructs. The full-length rat NCKX2 (NM_031743.2) was cloned into pcDNA3.1 $(+)$ plasmid (Invitrogen). The cytoplasmic loop region of NCKX2 (NCKX2-loop, amino acids 288-478) was isolated from fulllength NCKX2 and subcloned into the yeast expression vector pGBKT7 (Clontech) and the mammalian expression vector pcDNA3.1(+) with $\mathrm{N}$-terminal c-myc tag (myc-NCKX2-loop). NCKX2-GFP was constructed by inserting the EGFP coding sequence into the N-terminal BstEII restriction site of NCKX2 in the pcDNA3.1(+) vector. The NCKX2-FLAG construct was created by replacing the N-terminal 8 amino acids $\left({ }^{90}{ }^{D L N D K I R D}{ }^{97}\right)$ by the FLAG tag (DYKDDDDK). Mouse KIF4 (NM_008446.2), KIF5B (NM_008448.3), KIF21A (NM_001109042.1), and KIF21B (NM_001039472.1) cDNAs were obtained by RT-PCR from total mouse brain RNA and ligated into the mammalian expression vector pFLAG-CMV2 (Sigma-Aldrich). The dominant-negative kinesin (dnKIF) constructs were generated by deleting motor domains and placing the resulting constructs into the pFLAGCMV2 and yeast expression vector pGADT7 (Clontech). For dnKIF21A, amino acids 1-1231 were deleted; for dnKIF21B, amino acids 1-1301 were deleted; for dnKIF4, amino acids 1-350 were deleted; and for dnKIF5B, amino acids 1-330 were deleted. C-terminal EGFP-tagged synaptophysin (Syp-GFP) was cloned into pcDNA3.1(+). The wild-type and mutant (K44A) dynamin-1 cloned into pEGFP-C1 (Clontech) were gifts from Dr. S. Chang (Seoul National University, Seoul, South Korea). pDsRed2-N1 (Clontech) was used for visualizing the neurites of a cultured neuron.

Yeast two-hybrid screening. Yeast two-hybrid screening for proteins interacting with the NCKX2-loop was performed with the MATCHMAKER System (Clontech) according to the user manual. The yeast strain AH109 containing the pGBKT7-NCKX2-loop as bait plasmid was mated with the yeast strain Y187 pre-transformed with a cDNA library $\left(\sim 10^{6}\right.$ clones) from mouse brain cloned into the vector pGADT7. Selection of positively interacting clones was based on their abilities to express HIS3 and ADE2 genes, which enabled the yeast to grow in the absence of histidine or both histidine and adenine at $30^{\circ} \mathrm{C}$. Positive clones were identified by sequencing. Positive interactions were confirmed by the growth of AH109 cotransformed with both pGBKT7-NCKX2-loop (bait) and pGADT7-dnKIF21A (prey) on the selective plates of synthetic dropout media. The combination of the p53-bait and SV40 large $\mathrm{T}$-antigen prey was used as a control for positive interaction.

Cell culture. Hippocampal neurons were cultured on coverslips suspended above an astrocyte feeder layer. The protocol for low-density neuron-glia coculture is described by Kaech and Banker (2006). All work with animals was conducted in accordance with the animal welfare guidelines of Seoul National University. In brief, 2 weeks before neuronal culture, astrocytes were obtained by passing a cortical cell suspension from postnatal day 1 (P1) Sprague Dawley (SD) rat of either sex through a cell strainer (40 $\mu \mathrm{m}$ mesh; Falcon; BD Biosciences Discovery Labware), and then cultured in glial medium [minimum essential medium (MEM) (Invitrogen) supplemented with $0.6 \%$ glucose, $1 \mathrm{~mm}$ pyruvate, $2 \mathrm{~mm}$ GlutaMAX-I (Invitrogen), 10\% horse serum (HS) (Invitrogen), and 1\% penicillin-streptomycin (PS) (Invitrogen)]. Hippocampi from embryonic day 18 (E18) SD fetal rats were dissected in HBSS (Invitrogen) and digested with papain (Worthington), and then triturated with a polished half-bore Pasteur pipette. The neurons in plating medium [the same composition with glial medium except for $10 \%$ fetal bovine serum (FBS) (Invitrogen) instead of HS] were plated on poly-D-lysine (SigmaAldrich)-coated glass coverslips in a $60 \mathrm{~mm}$ culture dish at a density of $0.7-1.4 \times 10^{4}$ cells $/ \mathrm{cm}^{2}$. Paraffin dots ("feet" to suspend the coverslips above the glial feeder layer) were previously applied to the coverslips. The next day, coverslips were transferred above the glial culture preincubated in Neurobasal A medium (Invitrogen) supplemented with $0.5 \mathrm{~mm}$
GlutaMAX-I and 2\% B-27 supplement (Invitrogen) for $1 \mathrm{~d}$. To prevent proliferation of glial cells, $5 \mu \mathrm{M} 1-\beta$-D-cytosine-arabinofuranoside (Sigma-Aldrich) was added at the fourth day in vitro (DIV4). HEK293 cells (ATCC) were plated at a density of $5 \times 10^{4}$ cells per $100 \mathrm{~mm}$ culture dishes and maintained in DMEM (Invitrogen) supplemented with $10 \%$ FBS and $1 \%$ PS.

Transfection. Primary hippocampal neurons (DIV3-DIV6) were transfected using calcium phosphate (Ryan et al., 2005). Before transfection, the conditioned culture medium was saved and neurons were incubated with $2 \mathrm{ml}$ of Neurobasal A containing 25 mM HEPES, pH 7.3 (adjusted with $\mathrm{NaOH}$ ). The DNA/calcium phosphate precipitate was prepared by mixing 1 vol of DNA (up to $15 \mu \mathrm{g}$ ) in $250 \mathrm{mM} \mathrm{CaCl}_{2}$ with an equal volume of $2 \times \mathrm{HBS}(280 \mathrm{~mm} \mathrm{NaCl}, 50 \mathrm{~mm}$ HEPES, $1.5 \mathrm{~mm}$ $\mathrm{Na}_{2} \mathrm{HPO}_{4}$, $\mathrm{pH}$ 7.1) using a vortex mixer. Then, $200 \mu \mathrm{l}$ of DNA/calcium phosphate mixture was added dropwise to the cultured neurons, and neurons were incubated at $37^{\circ} \mathrm{C}$ for $15 \mathrm{~min}$. After the incubation, DNA/ calcium phosphate precipitates were washed out three times with fresh Neurobasal A for $5 \mathrm{~min}$, and the cells were returned to the saved original medium. For Figure 3, NCKX2-GFP and DsRed with or without either of KIF21A, dnKIF21A, or dnKIF21B were transfected to hippocampal neurons. NCKX2-GFP and either of the three kinesin constructs were cotransfected in a ratio of 1:4. NCKX2-FLAG and wild-type or mutant dynamin- 1 constructs were cotransfected in a ratio of 1:1. HEK293 cells were also transfected using calcium phosphate. The procedures were essentially the same except the medium was not changed before and after adding of DNA/calcium phosphate mixture to the culture.

Antibodies for NCKX2 and KIF21A. For NCKX2, we used two different kinds of antibodies denoted by anti-NCKX $2_{\text {ext }}$ and anti-NCKX2 $2_{\text {loop }}$. The former is a polyclonal antibody raised against the extracellular epitope (amino acids 90-102) of the rat NCKX2 (Thermo Fisher Scientific/Affinity BioReagents). We raised the latter antibody (anti-NCKX2 $2_{\text {loop }}$ ) against purified His6-tagged cytoplasmic region of NCKX2 (His6NCKX2-loop; amino acids 288-478). The expression of His6-NCKX2loop was induced by $0.1 \mathrm{~mm}$ isopropyl thio- $\beta$-D-galactopyranoside (Fluka) in Escherichia coli strain BL21 (DE3) and purified by affinity column (QIAGEN). Immunizing and sampling of rabbit antisera were performed by a commercial facility (AbFrontier). The anti-KIF21A antibody was raised against glutathione $S$-transferase fused KIF21A (amino acids 1101-1213). Using the same procedure as NCKX2, we obtained two different anti-KIF21A antibodies from two different rabbits that were immunized with the same immunogen.

Coimmunoprecipitation. Hippocampal neurons from E18 SD rats were plated at a density of $4.5 \times 10^{4}$ cells $/ \mathrm{cm}^{2}$ on $100 \mathrm{~mm}$ culture plates coated with $100 \mu \mathrm{g} / \mathrm{ml}$ poly-D-lysine and cultured for 2 weeks. The neurons at DIV14 were washed once with Dulbecco's PBS (DPBS) (Invitrogen) and lysed for $30 \mathrm{~min}$ on ice in lysis buffer containing $25 \mathrm{~mm}$ Tris $(\mathrm{pH}$ 7.4 adjusted with $\mathrm{HCl}$ ), $10 \mathrm{~mm} \mathrm{NaCl}, 1 \%$ sodium cholate hydrate, $1 \%$ Triton X-100 (v/v), $1 \mathrm{~mm}$ phenylmethyl sulfonyl fluoride (SigmaAldrich), and $0.2 \%$ protease inhibitor mixture (Sigma-Aldrich) (Oh et al., 2006; Hong et al., 2009). Cell lysates were then centrifuged at $8200 \times$ $g$ for $15 \mathrm{~min}$ at $4^{\circ} \mathrm{C}$, and the protein concentrations were determined by Bradford protein assay (Bio-Rad). The supernatants containing $500 \mu \mathrm{g}$ of total proteins were incubated with rabbit anti-KIF21A IgG or rabbit anti-NCKX $2_{\text {loop }}$ IgG immobilized on the amine-reactive gel according to the manufacturer's instructions (ProFound coimmunoprecipitation kit; Thermo Fisher Scientific/Pierce). Nonimmune rabbit IgG (Calbiochem) was used as control. The immunoprecipitated protein complexes were subjected to SDS-PAGE and Western blot analysis with anti-NCKX2 $2_{\text {ext }}$ and anti-KIF21A antibodies.

HEK293 cells were seeded in $100 \mathrm{~mm}$ culture dishes at $\sim 70 \%$ confluence and transfected with myc-NCKX2-loop and FLAG-tagged KIF variants, either KIF21A, KIF21B, dnKIF21A, or dnKIF21B, using calcium phosphate. After culture for $20-48 \mathrm{~h}$, the cells were washed twice with DPBS, solubilized in ice-cold lysis buffer containing $20 \mathrm{~mm}$ Tris ( $\mathrm{pH} 7.4$ adjusted with $\mathrm{HCl}$ ), $140 \mathrm{~mm} \mathrm{NaCl}, 10 \%$ sucrose, 1 mM EDTA, $1 \mathrm{~mm}$ $\mathrm{Na}_{3} \mathrm{VO}_{4}, 1 \% \mathrm{NP}-40(\mathrm{v} / \mathrm{v})$, and $0.2 \%$ protease inhibitor mixture. Cell lysates were clarified by centrifugation at $8200 \times g$ for $15 \mathrm{~min}$ at $4^{\circ} \mathrm{C}$. The supernatants were incubated with anti-c-myc antibody-conjugated agarose beads (Sigma-Aldrich) for $1 \mathrm{~h}$. The beads were then washed three 
times with lysis buffer for $5 \mathrm{~min}$, and the immunoprecipitated protein complexes were denatured in $2 \times$ SDS sample buffer and subjected to SDS-PAGE and Western blot analysis with anti-FLAG.

Western blotting. Cell lysates or immunoprecipitated proteins were separated by SDS-PAGE and transferred onto a polyvinylidene difluoride membrane (Millipore). The resulting blots were blocked for $1 \mathrm{~h}$ in PBS plus $0.1 \%$ Triton X-100 (0.1\% PBST) containing 5\% skim milk (Difco). The blots were incubated overnight at $4^{\circ} \mathrm{C}$ with specific primary antibodies: rabbit polyclonal anti-NCKX2 $2_{\text {ext }}$ (1:100; Thermo Fisher Scientific/ Affinity BioReagents), rabbit polyclonal anti-KIF21A (1:500), mouse monoclonal anti-FLAG M2 (1:5000; Sigma-Aldrich), mouse monoclonal anti-c-myc (1:500; Santa Cruz Biotechnology), or mouse monoclonal anti- $\beta$-actin (1:2500; Abcam) as loading controls. After washing three times, the blots were incubated at room temperature (RT) for $1 \mathrm{~h}$ with the corresponding horseradish peroxidase-conjugated secondary antibodies: goat anti-rabbit IgG (1:2000; Abcam) or goat anti-mouse IgG (1: 5000; Jackson ImmunoResearch). After washing three times, detection was performed using enhanced chemiluminescence reagent (GE Healthcare Bioscience). The membranes were then exposed to x-ray films (Agfa Gevaert). Films were digitally scanned and signals were quantified using the densitometric analysis software Multi Gauge (Fujifilm). For washing the immunoblots, PBS containing $1 \%$ NP-40 and $0.1 \%$ SDS or $0.1 \%$ PBST was used.

For antibody preabsorption test, before Western blotting, each primary antibody (diluted in $0.1 \%$ PBST) was preincubated at RT for $2 \mathrm{~h}$ with $50 \mu \mathrm{g} / \mathrm{ml}$ of an appropriate antigen: affinity-purified protein or synthesized peptides. The antigen purification procedures for anti$\mathrm{NCKX}_{2}$ loop and anti-KIF21A are described above. The antigen peptide (amino acids 90-102; DLNDKIRDYTPQP) for anti-NCKX2 $2_{\text {ext }}$ antibody was synthesized by a commercial facility (AnyGen). To enhance the antigenicity of the peptide, we used the synthesized peptides with crosslinked to polystyrene beads (BeadTech) as an antigen, and thus bare polystyrene beads as control in Figure $1 G$.

Immunocytochemistry. For detecting total (both cytosolic and surface) protein, the cells were fixed with ice-cold $4 \%$ paraformaldehyde (PFA) or $3.8 \%$ formaldehyde in PBS for 20 min, washed with PBS, and then permeabilized with $0.1 \%$ PBST for 5 min at RT. The cells were further washed in PBS and blocked in blocking solution (5\% donkey or goat serum in $0.1 \%$ PBST) for $1 \mathrm{~h}$ at RT. Primary antibodies were diluted in blocking solution and incubated with samples for $1 \mathrm{~h}$ at RT or overnight at $4^{\circ} \mathrm{C}$. Primary antibody dilutions were as follows: rabbit polyclonal anti-NCKX2 $2_{\text {ext }}$ (1:100), mouse monoclonal anti-FLAG M2 (1:2500), mouse monoclonal anti-synaptophysin (1:100; Sigma-Aldrich), mouse monoclonal anti-Tau-1 (1:500; Sigma-Aldrich), mouse monoclonal anti-microtubule-associated protein 2 (MAP2) (1:500; Millipore Bioscience Research Reagents), and rat monoclonal anti-neurofilament $\mathrm{H}$ (NF-H) (1:500; Millipore Bioscience Research Reagents). After three washes in PBS, cells were incubated with secondary antibodies diluted in blocking solution for $1 \mathrm{~h}$ at RT in the dark. Secondary antibodies were used as follows: Alexa Fluor 488-conjugated goat anti-rabbit IgG (1:400 dilution; Invitrogen) for immunostaining of endogenous NCKX2; Cy5conjugated donkey anti-rabbit IgG (1:400; Millipore Bioscience Research Reagents) for NCKX2-GFP;rhodamine-conjugated donkey anti-mouse IgG (1:200; Jackson ImmunoResearch) for NCKX2-FLAG, FLAGKIF21A (see Fig. 2), and MAP2 (see Figs. 6B, 7A); Cy5-conjugated donkey anti-mouse IgG (1:200; Jackson ImmunoResearch) for NCKX2FLAG (see Fig. 7B), MAP2 (see Figs. 3, 4, 6A, 7E), Tau-1 (see Figs. 3, 6A), and synaptophysin (see Fig. 6A); for NF-H, Alexa Fluor 568-conjugated goat anti-rat IgG (1:400; Invitrogen; see Fig. 7) and Cy5-conjugated goat anti-rat IgG (1:500; Jackson ImmunoResearch; see Fig. 2) were used. Finally, the cells were washed three times in PBS and mounted with fluorescent mounting medium (Dako). In Figure $2 \mathrm{~A}$, nuclear staining by DAPI was performed to visualize untransfected cells. Before mounting, cells were incubated with DAPI in PBS (1:5000; Sigma-Aldrich) for $5 \mathrm{~min}$ and washed with PBS, and then mounted.

For surface immunostaining of NCKX2, live cells were incubated with rabbit anti-NCKX2 ${ }_{\text {ext }}(1: 100)$ or rabbit anti-GFP (1:100; Millipore Bioscience Research Reagents) in serum-free culture medium for $15 \mathrm{~min}$ at $36^{\circ} \mathrm{C}$ or at $4^{\circ} \mathrm{C}$, rinsed with culture medium, fixed with ice-cold $4 \%$ PFA or 3.8\% formaldehyde in PBS for 20 min, and washed with PBS. Then cells were incubated with secondary antibodies diluted in serum-free culture medium for $30 \mathrm{~min}$. For double immunostaining with MAP2, Tau-1, synaptophysin, or NF-H, cells labeled with anti-NCKX2 $2_{\text {ext }}$ antibody and then fixed were subsequently permeabilized with $0.1 \%$ PBST for $5 \mathrm{~min}$ at RT. The fixed cells were incubated in blocking solution for $1 \mathrm{~h}$ at RT, and then with anti-MAP2, Tau-1, synaptophysin, or NF-H antibodies diluted in blocking solution (Bel et al., 2009). Subsequent steps were the same as those for total protein immunostaining. The immunostained cells were imaged with FV300 (Olympus) or TCS-SP2 (Leica) confocal laser-scanning microscopes with 60 or $63 \times$ waterimmersion objectives, and then processed using Fluoview or Leica Lite.

Quantitative analysis of colocalization. We quantified the colocalization of NCKX2 with FLAG-KIF21A by intensity correlation analysis on the NF-H-immunoreactive axonal neurite of a DIV12 cultured hippocampal neuron, which were triple-immunostained with antiNCKX2 $2_{\text {ext }}$ (green), anti-FLAG (red), and anti-NF-H (blue). The region of interest (ROI) was defined as a binary mask covering the axonal neurite of a neuron expressing FLAG-KIF21A. It was made from the NF-H fluorescence image of the FLAG-positive neuron using the "ImageSeedFill" routine supplied by Igor Pro (version 4.1; Wavemetrics). This routine makes a cell mask by taking a seed pixel and filling a region defined by all contiguous pixels whose pixel values are higher than a given threshold value. After subtraction of background fluorescence, we analyzed paired green $\left(G_{i}\right)$ and red $\left(R_{i}\right)$ fluorescence intensities of each pixel within the ROI. The value for the product of difference from the mean (PDM) at the ith pixel was calculated according to the following equation: $\mathrm{PDM}_{\mathrm{i}}=\left(G_{i}-g\right) / G_{\max } \times\left(R_{i}-r\right) / R_{\max }$, where $g$ and $r$ are the mean values of green and red fluorescence intensities in the ROI, respectively; $G_{\max }$ and $R_{\max }$ are the maximal green and red intensities in the ROI, respectively. If the paired green and red fluorescence intensities vary together in an image, the number of pixels having a positive PDM value will be larger than $50 \%$ of total pixels, because a pixel above (below) the average in the one channel will be above (below) the average in the other. The intensity correlation quotient (ICQ) was calculated as follows: ICQ $=$ (the number of positive PDM pixels/the total number of pixels in the ROI -0.5 . The ICQ value is expected to be zero for random staining, $0<$ ICQ $<0.5$ for dependent staining, and $-0.5<$ ICQ $<0$ for segregated staining. We tested whether a ICQ value is significantly different from zero using the normal approximation of the sign test ( $\mathrm{Li}$ et al., 2004).

Determination of dendritic and axonal fluorescence of NCKX2. To determine the fluorescence ratio of axonal-to-dendritic compartment (ADR) in a neuron, we made a binary mask image of a dendrite or an axon using the "ImageSeedFill" routine of Igor Pro. After a dendritic mask was made from a MAP2 image (see Figs. 3I, 4E, middle for an exemplar mask image), an axon image was obtained by setting the dendritic pixels (defined by the MAP2 mask) on the NCKX2 image to a zero value (see Figs. 3I, $4 E$, right). To eliminate the background fluorescence of cell-free regions, we subtracted the mean background gray value from the image, and removed salt-and-pepper type background noise by convoluting the image with a $3 \times 3$ median filter. Finally, we defined ROIs along a dendritic or an axonal compartment on the processed image and determined ADR from the averaged fluorescence count of nonzero pixels in the axonal and dendritic ROIs.

A few seed pixels on the cell were enough for the mask covering a MAP2 fluorescence image (see Figs. 3, 4), and thus they were manually set in Igor Pro. To make a cell mask from the image of NCKX2-GFP or EGFP-tagged dynamin-1, which exhibits a speckled fluorescence pattern (see Fig. 7), we took a set of seed pixels from the pixels on the traced line drawn on the neurites of the same image using NeuronJ and fed the seed pixels into the "ImageSeedFill" routine of Igor Pro. The consequent cell mask made from an image of EGFP fluorescence covered the entire cellular region expressing NCKX2-GFP or NCKX2-FLAG. We determined the dendritic surface expression level from the mean gray value of pixels on the mask of the MAP2-positive neurites. The axonal surface expression level was determined from the MAP2-negative neurite.

Hippocampal organotypic slice culture. Slice cultures were prepared from $\mathrm{P} 6-\mathrm{P} 8 \mathrm{SD}$ rat pups of either sex mostly according to the procedure 
described by De Simoni and Yu (2006) according to the animal welfare guidelines of Seoul National University. Briefly, hippocampal slices (thickness, $350 \mu \mathrm{m}$ ) were obtained with vibratome (DTK-1000 ZERO 1; Dosaka) and placed on a porous $(0.4 \mu \mathrm{m})$ membrane (Millicell-CM; Millipore). The culture medium was a mixture of 50\% MEM, 25\% HS, $24 \%$ Eagle's buffered salt solution, and 1\% PS. Glucose was added to reach a final concentration of $36 \mathrm{~mm}$. After $4 \mathrm{~d}$ in culture, the medium was changed to serum-free Neurobasal medium with 2\% B-27 supplement, $1 \%$ GlutaMAX-I, 1\% PS, and 5 mm glucose added. The medium was changed every $2 \mathrm{~d}$.

Lentiviral transduction system and RNA interference. We used a lentiviral delivery system to knockdown NCKX2 or KIF21A. NCKX2targeting short-interfering RNA (siRNA) sequences ( $5^{\prime}$-ACAACAGTCT CATGAGGAA-3' for shNCKX2-1; 5'-GCTTCAATTCTCCACAAG A-3' for shNCKX2-2) and KIF21A-targeting siRNA sequence $\left(5^{\prime}\right.$ CGAGATCATCAACTTAGAC-3') were designed using the siRNA software tool available at the Whitehead Institute for Biomedical Research (http://www.whitehead.mit.edu). The luciferase-targeting siRNA sequence (5'-TAAGGCTATGAAGAGATAC-3') was used as a nontargeting siRNA control (Dharmacon). The synthesized NCKX2, KIF21Atargeting or nontargeting short-hairpin RNA (shRNA) oligonucleotides (CosmoGenetech) were ligated into the lentiviral vector pLentiLox3.7 (pLL3.7) (Rubinson et al., 2003), which could coexpress EGFP or monomeric RFP (mRFP). Lentivirus was produced by the commercial lentiviral packaging service (Macrogen). Lentiviruses in culture medium were filtered through $0.45 \mu \mathrm{m}$ filters (Satorius) and then concentrated with Amicon Ultra-4 centrifugal filter unit, MWCO $100 \mathrm{kDa}$ (Millipore), and aliquots were stored at $-80^{\circ} \mathrm{C}$ (Braam et al., 2008; Hioki et al., 2009). The efficiency of gene silencing was assessed in HEK293 cells and cultured hippocampal neurons by immunoblot analysis or immunocytochemistry. Lentiviral stocks in the presence of fresh $4 \mu \mathrm{g} / \mathrm{ml}$ polybrene (Davis et al., 2004) were delivered to DIV7-DIV8 organotypic cultured hippocampal dentate gyrus by local injection using a $10 \mu \mathrm{m}$ pore glass pipette (Davis et al., 2004). Primary cultured hippocampal neurons (DIV1DIV3) were infected with shRNA lentivirus at a multiplicity of infection of $\sim 1$.

Recording of reverse-mode NCKX current. The NCKX activity of NCKX2-GFP was tested by recording $\mathrm{Ca}^{2+}$ - and $\mathrm{K}^{+}$-dependent reversemode exchange current in the HEK293 cell perfused with high- $\mathrm{Na}^{+}$ internal pipette solution using whole-cell patch-clamp technique. NCKX2 activity was identified by dependence of outward current at the holding potential of $0 \mathrm{mV}$ on extracellular $\mathrm{Ca}^{2+}$ plus $\mathrm{K}^{+}$. The procedure for recording reverse-mode (outward) NCKX currents was previously described in detail in J. Y. Lee et al. (2006). In brief, whole-cell patchclamp recordings were attained on the HEK293 cells expressing NCKX2GFP with a pipette solution containing the following (in $\mathrm{mM}$ ): 120 $\mathrm{Na}$-gluconate, $10 \mathrm{KCl}, 20 \mathrm{HEPES}, 1 \mathrm{EGTA}, 4 \mathrm{MgATP}$, and $0.3 \mathrm{Na}_{2} \mathrm{GTP}$ at pH 7.2 (adjusted with $\mathrm{NaOH}$ ). Current recordings in baseline conditions were obtained while the cells were superfused with $\mathrm{Ca}^{2+}$ - and $\mathrm{K}^{+}$-free bath solution containing the following (in $\mathrm{mm}$ ): $140 \mathrm{LiCl}$, 0.5 EGTA, 1 $\mathrm{MgCl}_{2}, 10$ HEPES, and 10 glucose at $\mathrm{pH} 7.4$ (adjusted with LiOH). Reverse mode of NCKX currents was induced by bath application of test solution. $\mathrm{Ca}^{2+}$ plus $\mathrm{K}^{+}$(or $\mathrm{Ca}^{2+}$ plus $\mathrm{Cs}^{+}$) test solution contained 140 $\mathrm{mm} \mathrm{KCl}$ (or $140 \mathrm{~mm} \mathrm{CsCl}$ ) and $1 \mathrm{~mm} \mathrm{CaCl}_{2}$ instead of $140 \mathrm{~mm} \mathrm{LiCl}$ and 0.5 mM EGTA. $\mathrm{Ca}^{2+}$ test solution contained $1 \mathrm{mM} \mathrm{CaCl}_{2}$ instead of 0.5 mM EGTA.

Recording of $\mathrm{Ca}^{2+}$ transient in axon terminals of hippocampal granule cells. Cultured hippocampal slices were placed in a submerged recording chamber and perfused with artificial CSF (ACSF) composed of the following (in mM): $124 \mathrm{NaCl}, 26 \mathrm{NaHCO}_{3}, 3.2 \mathrm{KCl}, 2.5 \mathrm{CaCl}_{2}, 1.3 \mathrm{MgCl}_{2}$, $1.25 \mathrm{NaH}_{2} \mathrm{PO}_{4}$, and 10 glucose with $\mathrm{pH}$ adjusted at 7.4 by saturating with carbogen $\left(95 \% \mathrm{O}_{2}, 5 \% \mathrm{CO}_{2}\right)$. For calcium imaging, whole-cell patch clamp was attained on the mRFP-expressing soma of a dentate granule cell (GC) under visual control using differential interference illumination in an upright microscope (BX51WI; Olympus). The expression of mRFP was detected using an HcRed1 filter set (part no. 41043; Chroma). The whole-cell recordings were made using an EPC-10 amplifier (HEKA Elektronik) with a pipette solution containing the following (in mM): 140 K-gluconate, 5 di-Tris-phosphocreatin, $5 \mathrm{NaCl}, 4 \mathrm{MgATP}, 0.4 \mathrm{Na}_{2} \mathrm{GTP}$,
15 HEPES, 2.5 Na-pyruvate, $\mathrm{pH}$ 7.3, adjusted with $\mathrm{KOH}$, together with $200 \mu \mathrm{M}$ Oregon Green 488 BAPTA-5N (OGB-5N) $\left(K_{\mathrm{D}} \sim 50 \mu \mathrm{M}\right)$ or 50 $\mu \mathrm{M}$ Oregon Green 488 BAPTA-1 (OGB1) $\left(K_{\mathrm{D}} \sim 200 \mathrm{nM}\right)$ (Invitrogen). Both $\mathrm{Ca}^{2+}$ indicators were excited at $488 \mathrm{~nm}$. Calcium imaging was performed using a confocal laser-scanning system (FV300; Olympus) and a $60 \times$ water-immersion objective (numerical aperture, 0.9; LUMPlanFl/IR; Olympus). $\mathrm{Ca}^{2+}$ transients (CaTs) at hippocampal mossy fiber en passant boutons, somata, dendritic spines, and shafts of dentate granule cells were evoked by applying high-frequency stimulation, a train of short depolarizing pulses ( $2 \mathrm{~ms}$ in duration) from -80 to $0 \mathrm{mV}$ at 33 or $100 \mathrm{~Hz}$ for $5 \mathrm{~s}$, via whole-cell patch pipette on the soma, and recorded in $X Y$ scan mode at $5 \mathrm{~Hz}$ for $20 \mathrm{~s}$. A single action potential-induced CaT (AP-CaT) was recorded in line-scan mode at $540 \mathrm{~Hz}$. Suprathreshold current pulse to evoke AP-CaT was typically $600 \sim 900$ pA for $12 \mathrm{~ms}$. The relative $\mathrm{Ca}^{2+}$ increment is presented as $\Delta F / F_{0}$, where $F_{0}$ is resting fluorescence intensity of OGB-5N or OGB-1 after subtraction of background fluorescence, and $\Delta F$ is the fluorescence increment from $F_{0}$.

Estimation of dendritic $\mathrm{Ca}^{2+}$ clearance in the hippocampal granule cells. Acute hippocampal slices (thickness, $300 \mu \mathrm{m}$ ) were prepared from P14P17 SD rats as described by D. Lee et al. (2007). The whole-cell recordings were made at the soma of a hippocampal GC at $31-35^{\circ} \mathrm{C}$. The $\mathrm{K}^{+}$-based pipette solution contained the following (in $\mathrm{mm}$ ): $125 \mathrm{~K}$-gluconate, 20 $\mathrm{KCl}, 20$ HEPES, $5 \mathrm{Na}$-phosphocreatine, $4 \mathrm{MgATP}, 0.3 \mathrm{Na}_{2} \mathrm{GTP}$, and 0.1 fura- $4 \mathrm{~F}$ (Invitrogen) with $\mathrm{pH}$ adjusted at 7.3 using $\mathrm{KOH}$. For a $\mathrm{K}^{+}$-free pipette solution (denoted as $\mathrm{TMA}^{+}$-based pipette solution), K-gluconate and $\mathrm{KCl}$ were replaced with equimolar tetramethylammonium (TMA)gluconate and tetraethylammonium (TEA)-Cl, respectively. For low $\left[\mathrm{Na}^{+}\right]$ACSF, $125 \mathrm{~mm} \mathrm{NaCl}$ was replaced with equimolar choline-Cl. Serial images of fura- $4 \mathrm{~F}$ fluorescence were taken using a monochromator (Polychrome-IV; TILL Photonics) and an air-cooled slow-scan CCD camera (SensiCam; PCO). We used the built-in on-chip binning $(8 \times 16$ pixels) function to accelerate the frame rate $(40 \mathrm{~Hz}$; exposure time, $5 \mathrm{~ms})$. In the off-line analysis, an ROI including the proximal dendrite was drawn on the fluorescence image of a GC, and the ratio $\left(r=F_{\text {iso }} / F_{380}\right)$ of averaged fluorescence over the ROI at the isosbestic wavelength $(360 \mathrm{~nm}$; $\left.F_{\text {iso }}\right)$ to that at $380 \mathrm{~nm}\left(F_{380}\right)$ was converted to $\left[\mathrm{Ca}^{2+}\right]_{\mathrm{i}}$ according to the following equation: $\left[\mathrm{Ca}^{2+}\right]_{\mathrm{i}}=K_{\mathrm{eff}} \cdot\left(R-R_{\min }\right) /\left(R_{\max }-R\right)$, where $K_{\text {eff }}$ was estimated as $8.12 \mu \mathrm{M}$. Calibration parameters were determined by "in-cell" calibration as described previously (J. S. Lee et al., 2008).

To quantify $\mathrm{Ca}^{2+}$ clearance, we analyzed the decay phase of a CaT evoked by a short depolarizing pulse under different conditions. Because $\mathrm{Ca}^{2+}$ clearance depends on the peak $\Delta\left[\mathrm{Ca}^{2+}\right]$ level, the duration of depolarization was adjusted in the range between 50 and $100 \mathrm{~ms}$, such that the peak $\Delta\left[\mathrm{Ca}^{2+}\right]$ level of the evoked CaT is typically $1 \mu \mathrm{M}$. The decay phase was fitted with a biexponential function: $A_{0}+$ $A_{1} \cdot \exp \left(-r_{1} \cdot t\right)+A_{2} \cdot \exp \left(-r_{2} \cdot t\right)$. Assuming that intracellular $\mathrm{Ca}^{2+}$ buffers are alike among GCs, the weighted average of the rate constants $\left(r_{\mathrm{w}}\right)$, which is defined as $\left(A_{1} \cdot r_{1}+A_{2} \cdot r_{2}\right) /\left(A_{1}+A_{2}\right)$, can be regarded as a parameter representing $\mathrm{Ca}^{2+}$ clearance at the peak of the $\mathrm{CaT}$, and exhibits little dependence on the peak $\Delta\left[\mathrm{Ca}^{2+}\right]$ level when it is $>0.8 \mu \mathrm{M}$ (S.-H. Lee et al., 2009).

Statistical analysis. Data were analyzed using Igor Pro and are presented as mean \pm SEM. The statistical significance of differences between two experimental conditions was evaluated using Student's $t$ test using a significance level $(p)$ of 0.05 or 0.01 .

\section{Results}

\section{NCKX2 interacts with KIF21A}

NCKX2 has 11 proposed transmembrane segments (TMSs) and a large intracellular loop between the fifth and sixth TMSs (Cai et al., 2002; Kinjo et al., 2003) (Fig. 1A). We hypothesized that the cytosolic loop of NCKX2 interacts with a transporter molecule that mediates the axonal transport of NCKX2. To identify proteins interacting with NCKX2, we performed yeast two-hybrid screening of a mouse brain cDNA library ( $\sim 10^{6}$ clones $)$ using the central cytoplasmic loop of NCKX2 (NCKX2-loop; amino acids $288-478$ ) as bait. Sequence analysis revealed that two different positive clones (amino acids 1475-1573, 1513-1573) encoded the 
A

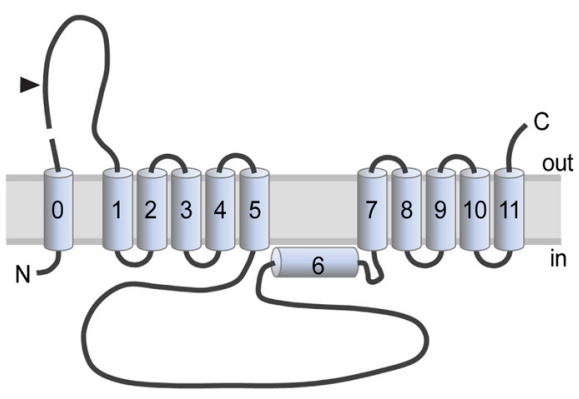

C

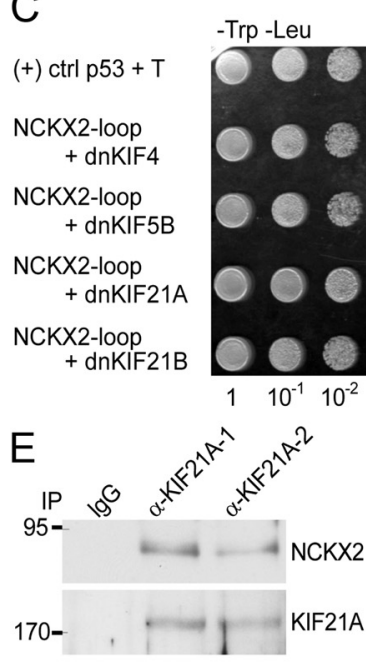

F Anti-NCKX2loop antibody

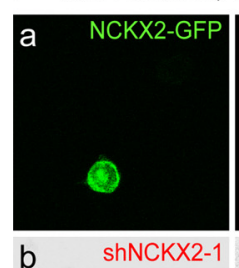

b

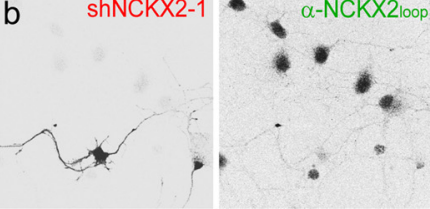

-Trp -Leu -His

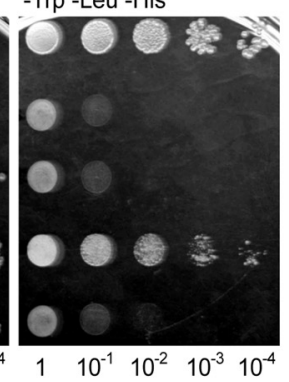

B

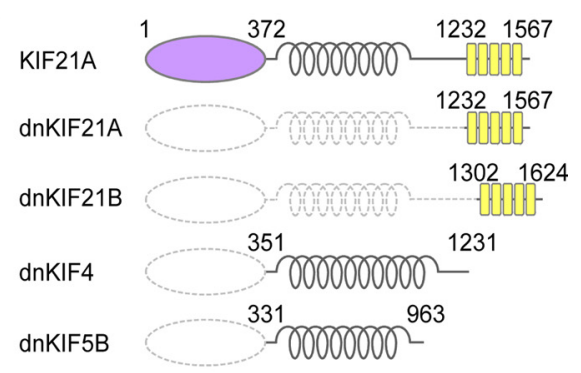

D

TF
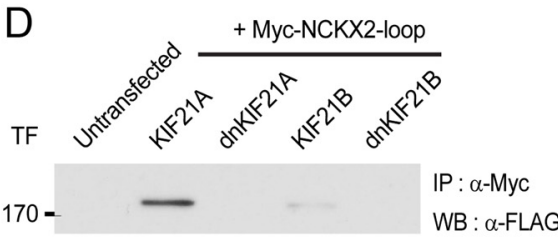

$40-$

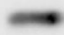

Input (lysates before IP)

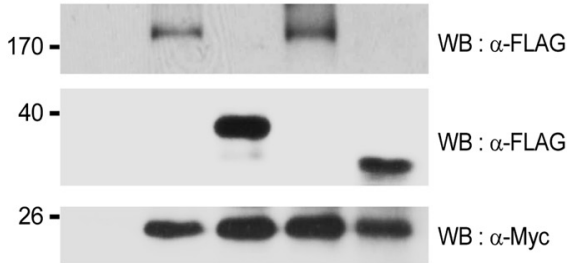

Figure 1. The intracellular loop of NCKX2 (NCKX2-loop) specifically interacts with the WD-40 repeats of KIF21A. A, A topological model of NCKX2. NCKX2 has a large intracellular loop between the fifth and sixth TMSs. The arrowhead indicates the position where EGFP was inserted to make EGFP-tagged NCKX2 (NCKX2-GFP). B, Schematic illustration of the structure of wild-type KIF21A and dominant-negative mutant (dn-) of KIF21A, KIF21B, KIF4, and KIF5B. The kinesin motor heads, coiled-coil stalks, and WD-40 repeats are shown by the violet oval, gray-colored coils, and yellow boxes, respectively. Deleted regions are indicated by dotted lines. Note that wild-type KIF4 and KIF5B tails have no WD-40 repeats. C, The yeast two-hybrid reporter strain AH109 transformed with the pGBKT7-NCKX2-loop and pGADT7-KIF variants (dnKIF4, dnKIF5B, dnKIF21A, or dnKIF21B) was grown overnight in liquid selective synthetic dropout medium (-Trp-Leu) and 10-fold serial dilutions were spotted on the selective synthetic dropout medium -Trp-Leu or-Trp-Leu-His plates. The combination of the T-antigen prey and p53 bait was used as a control for positive interaction. D, KIF21A and dnKIF21A but little KIF21B and dnKIF21B were coimmunoprecipitated with myc-NCKX2-loop (top), when expressed exogenously in HEK293 cells. KIF variants were tagged with FLAG. The loaded amounts of the different FLAG-tagged KIF constructs and myc-NCKX2-loop in each of the transfected cell lysates before coimmunoprecipitation are shown in the bottom panel. $\boldsymbol{E}$, Endogenous NCKX2 was coimmunoprecipitated with KIF21A using two different kinds of anti-KIF21A antibodies (left) and reciprocally endogenous KIF21A was coimmunoprecipitated with NCKX2 (right) from rat hippocampal neurons (at DIV14). Normal rabbit lgG was used as negative control. TF, Transfection; $\mathbb{I P}$, immunoprecipitation; WB, Western blot. $\boldsymbol{F}$, Reliability test of NCKX2 $2_{\text {loop }}$ antibody. Fa, Anti-NCKX2 $2_{\text {loop }}$ antibody (red) specifically immunolabeled the HEK293 cell expressing NCKX2-GFP (green). The untransfected cell is shown in the transmitted image. Scale bar, $20 \mu \mathrm{m}$. Fb. When endogenous NCKX2 was immunolabeled with anti-NCKX2 $2_{\text {loop }}$ (green), the NCKX2-depleted hippocampal neurons using shNCKX2 (Fig. 5) were not immunostained with anti-NCKX2 $2_{\text {loop. }}$. The shNCKX2-transfected cells were identified by coexpressing mRFP fluorescence (red). Scale bar, $50 \mu \mathrm{m}$. G, Preabsorption tests of anti-

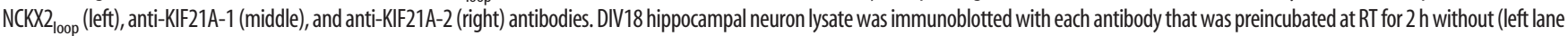
of each panel; control) or with (right lane of each panel) an appropriate antigen. $\beta$-Actin was detected as a loading control (bottom of each panel).

C-terminal segment of KIF21A, which contains WD-40 repeats, a putative cargo-binding domain (Marszalek et al., 1999). To verify the specific interaction of NCKX2 with KIF21A, we compared the interaction of NCKX2-loop with KIF21A to that with other KIF members using the yeast two-hybrid system. We created con- structs for cargo-binding domains of four different KIF members: KIF4 (same family as KIF21A), KIF5B (different family from KIF21A), KIF21A, and KIF21B, which are free of "motor" or "motor and stalk" domains (referred to as "dominantnegative" variants; Fig. 1B). NCKX2-loop and dominant- 
negative KIF21A (dnKIF21A) were cotransformed into a haploid yeast, AH109, resulting in growth of colonies on the triple dropout plate (-Trp, -Leu, -His). However, none of the other dominant-negative variants of KIF (dnKIF4, dnKIF5B, or dnKIF21B) allowed growth of colonies on the triple dropout plate when cotransformed together with NCKX2-loop (Fig. 1C). These data are indicative of high specificity in the interaction between NCKX2-loop and KIF21A.

To test whether KIF21A and NCKX2-loop interact in a mammalian cell environment, we transfected HEK293 cells with c-myc-tagged NCKX2-loop (myc-NCKX2-loop) plus one of FLAG-tagged KIFs (FLAG-KIFs) and performed coimmunoprecipitation. We found that myc-NCKX2-loop was coimmunoprecipitated with FLAG-KIF21A or FLAG-dnKIF21A, but little with FLAG-KIF21B or FLAG-dnKIF21B (Fig. 1D). These results indicate that the NCKX2-loop interacts specifically with the WD-40 repeats of KIF21A.

Next, we verified the association of the endogenous NCKX2 with KIF21A in neurons. The lysate of cultured hippocampal neurons was immunoprecipitated with two different kinds of anti-KIF21A antibody (for details, see Materials and Methods). NCKX2 was detected by immunoblotting in the anti-KIF21A immunoprecipitate (Fig. $1 E$, left). Conversely, KIF21A was detected in the immunoprecipitate brought down by anti-NCKX2 $2_{\text {loop }}$ (for its definition, see Materials and Methods; Fig. 1 E, right), indicating that endogenous NCKX2 and KIF21A are parts of a complex in a neuron. The specificity of anti-KIF21A and anti-NCKX2 antibodies was verified by immunocytochemistry (Fig. $1 F$ ) and/or by the antibody preabsorption tests (Fig. 1G).

To test further the hypothesis that NCKX2 is associated with KIF21A, we examined whether NCKX2 and KIF21A are colocalized in a neuron. We performed immunocytochemistry on cultured hippocampal neurons expressing FLAG-KIF21A. Triple immunostaining with anti-FLAG, anti-NCKX $2_{\text {ext }}$ (see Materials and Methods for its definition; Fig. $2 A, B$, for specificity test) and anti-NF-H revealed that endogenous NCKX2 was colocalized with FLAG-KIF21A at the axon (Fig. 2C,D). To quantify the colocalization between NCKX2 and FLAG-KIF21A, the correlation of fluorescence intensities was estimated by calculating the PDM in each pixel in the binary mask of the FLAG-positive axonal neurite (Fig. 2E) (for details, see Materials and Methods). Calculated PDM values are shown as a scattered plot of green and red pixel intensities against their PDM value at each paired pixel (total number of pixel pairs, 2056; Fig. 2F). The plot clearly skewed toward the positive PDM value, and this analysis generated the ICQ of +0.20 that was significantly different from zero $\left(P_{\text {sign test }}<0.001\right)$. The distribution of NCKX2/FLAG-KIF21A complexes along the axon could be clearly represented by the PDM image, an image in which each pixel is equal to the PDM value at that location (Fig. 2Eb). The line profiles of NCKX2 (green) and FLAG-KIF21A (red) fluorescence intensities as well as the PDM image demonstrate that the NCKX2/FLAG-KIF21A complexes are localized in puncta along the axon fiber and in the axon terminal (Fig. 2G).

\section{Axonal transport of NCKX2 is mediated by KIF21A}

We tested whether the interaction of NCKX2 with KIF21A is functionally relevant to the transport of NCKX2. To visualize NCKX2 in neurons, we created a NCKX2-GFP fusion protein by inserting EGFP into the extracellular domain of NCKX2, downstream of the $\mathrm{N}$-terminal signal sequence (Fig. $1 A$ ). Its functional competence was confirmed following expression in HEK293 cells
(Fig. 3A). When we expressed NCKX2-GFP in cultured rat hippocampal neurons, NCKX2-GFP was distributed throughout the cell including neurites (Fig. 3B, left). In contrast, when dnKIF21A was coexpressed together with NCKX2-GFP, little GFP signal was observed on the longest neurite, a putative axon fiber (Dotti et al., 1988) (Fig. 3B, right), implying that overexpressed dnKIF21A inhibits the axonal transport of NCKX2 by overriding endogenous KIF21A. We quantified the transport of NCKX2 by axon tracing. To this end, NCKX2-GFP was cotransfected with DsRed, a red fluorescent protein into primary cultured hippocampal neurons. Cell morphology was visualized from the fluorescence of DsRed. After tracing the longest neurite using NeuronJ, a neurite tracing program (Meijering et al., 2004), we measured the GFP intensity values on the traced line (Fig. $3 C, D$ ). In this experiment, we presumed the longest neurites as axons. Overexpression of dnKIF21A affected neither the length of axon fibers (Fig. $3 F$ ) nor the expression level of NCKX2-GFP judged from the GFP fluorescence at the soma (Fig. 3C-E). Compared with the neurons expressing NCKX2-GFP alone or NCKX2-GFP plus KIF21A, those expressing dnKIF21A displayed significantly lower fluorescence intensity of NCKX2-GFP along the axon fiber, suggesting that the axonal transport of NCKX2-GFP is inhibited by dnKIF21A (Fig. 3E). In contrast, dnKIF21B did not inhibit the axonal transport of NCKX2-GFP (Fig. 3E).

To confirm the effects of dnKIF21A on the axonal transport of NCKX2, dendrites and axon fibers were identified by immunochemical labeling with MAP2 or Tau-1, respectively. When NCKX2-GFP was coexpressed with KIF21A, NCKX2-GFP was localized at both the MAP2-positive neurites (dendrites) and the MAP2-negative neurite (axon) (Fig. 3G). When NCKX2-GFP was coexpressed with dnKIF21A, however, NCKX2-GFP overlapped with MAP2 but not with Tau-1 (Fig. $3 H$ ). To quantify the effect of dnKIF21A on the axonal transport of NCKX2-GFP, we calculated the axon-to-dendrite ratio (ADR) of NCKX2-GFP fluorescence. The dendritic and axonal NCKX2-GFP fluorescence levels were measured from the ROI that covers the MAP2positive or -negative neurite, respectively, after nullifying the background fluorescence (Fig. 3I) (for details, see Materials and Methods). The ADR of NCKX2-GFP was significantly reduced in the neurons coexpressing dnKIF21A compared with those coexpressing KIF21A (Fig. 3J; KIF21A, $0.914 \pm 0.059, n=12$; dnKIF21A, $0.474 \pm 0.078, n=9 ; p<0.01)$. In contrast, the fluorescence of NCKX2-GFP in the dendrites normalized to that in the soma [dendrite-to-soma ratio (DSR)] was not significantly different between KIF21A and dnKIF21A-expressed groups (Fig. 3j; KIF21A, $0.109 \pm 0.017, n=12$; dnKIF21A, $0.156 \pm 0.016, n=$ $9 ; p>0.05)$. These results confirm that dnKIF21A inhibited the axonal transport of NCKX2.

To verify that the effects of dnKIF21A are specific for NCKX2, we examined transport of another presynaptic protein, synaptophysin. dnKIF21A did not inhibit the axonal transport of GFPtagged synaptophysin (Fig. 3K), indicating that dnKIF21A does not cause nonspecific inhibition of axonal transport.

\section{Knockdown of KIF21A abolishes the axonal transport of NCKX2}

To test whether KIF21A mediates the axonal transport of endogenous NCKX2, we depleted KIF21A using shRNA. Cotransfection in HEK293 cells of shRNA targeted to KIF21A (shKIF21A) together with FLAG-KIF21A completely knocked down the expression of KIF21A (Fig. 4A). We used a lentiviral delivery system to knockdown the endogenous KIF21A in hippocampal neurons. Immunoblot analyses at different time 
A Anti-NCKX2ext antibody

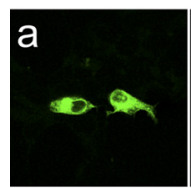

NCKX2-GFP

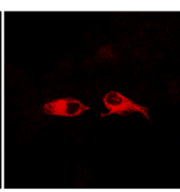

$\alpha-\mathrm{NCKX} 2 \mathrm{ex}$

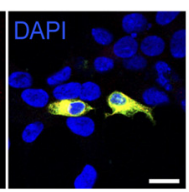

Merge

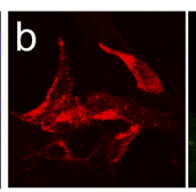

NCKX2-FLAG

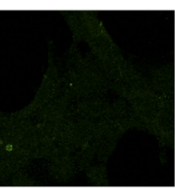

$\alpha-N C K X 2$ ext

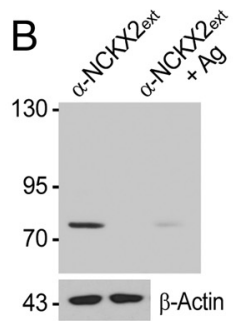

Merge

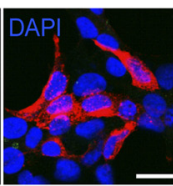

43- $-\beta$-Actin
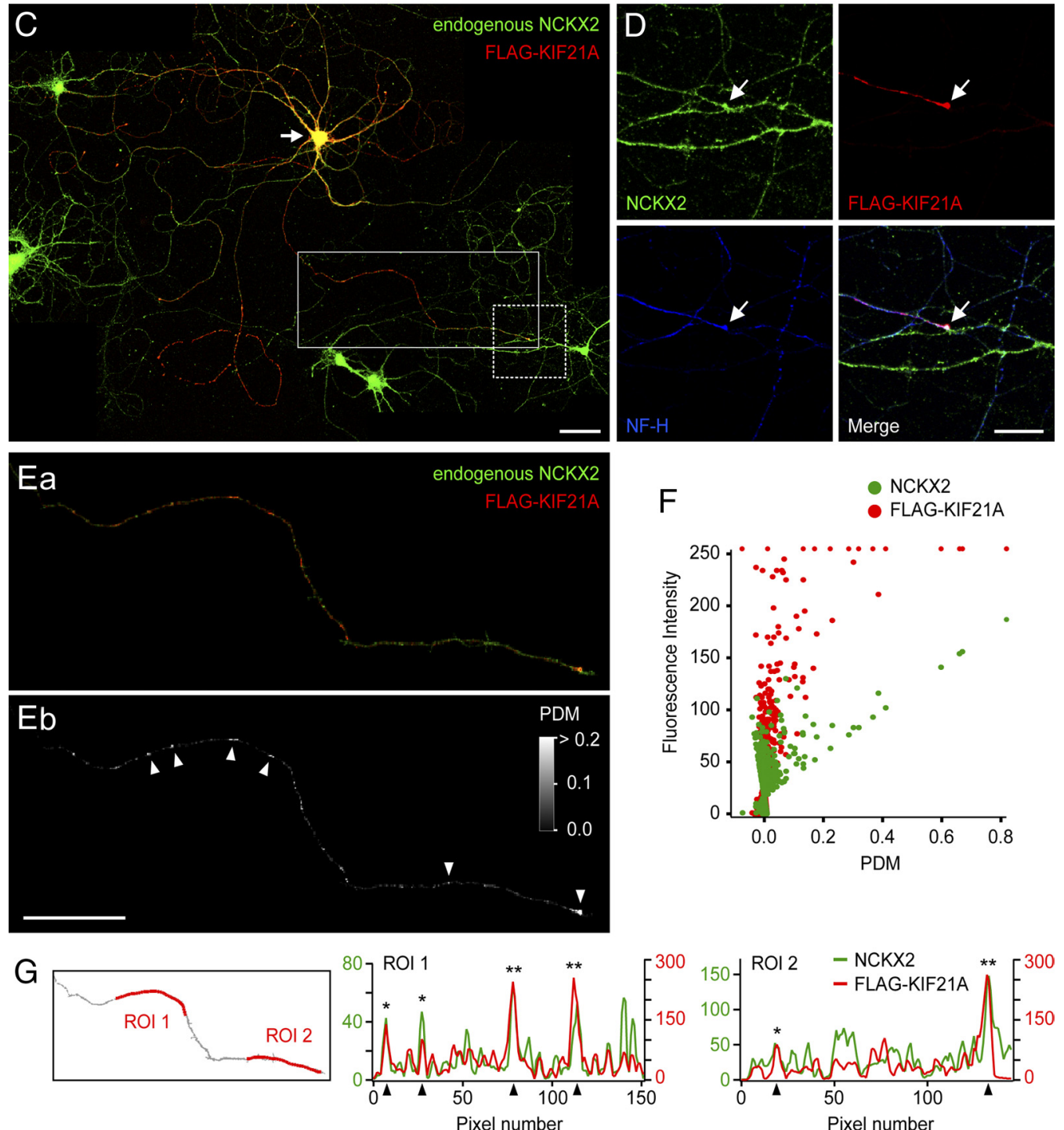

Figure 2. Colocalization of NCKX2 and KIF21A. A, Reliability test of NCKX2 ${ }_{\text {ext }}$ antibody. Aa, The HEK293 cells expressing NCKX2-GFP (green) were specifically labeled with anti-NCKX2 $2_{\text {ext }}$ antibody (red). Untransfected cells were visualized by DAPI staining (blue). Scale bar, $20 \mu \mathrm{m}$. $\boldsymbol{A} \boldsymbol{b}$, Because the anti-NCKX2 ext $_{\text {a }}$ antibody was raised againsta synthetic peptide within the N-terminal extracellular region (residues 90 -102), HEK293 cells expressing NCKX2-FLAG, in which the FLAGtag $\left({ }^{90}\right.$ DYKDDDD K ${ }^{97}$ ) replaces the N-terminal 8 amino acids of the epitope, were not immunoreactive to the anti-NCKX2 2 (green). Expression of NCKX2-FLAG was detected by anti-FLAG (red), and untransfected cells were visualized by DAPI staining (blue). Scale bar, $20 \mu \mathrm{m}$. B, Preabsorption test of anti-NCKX2 $2_{\text {ext }}$ antibody. DIV18 hippocampal neuron lysate was immunoblotted using anti-NCKX2 ${ }_{\text {ext }}$ antibody without (left lane) or with (right lane) preincubation of the antigen peptide. $\beta$-Actin was detected as a loading control (bottom panel). $C$, Endogenous NCKX2 (green) and transfected FLAG-KIF21A (red) were colocalized not only in the somatodendritic region but also in the axon terminals of the cultured hippocampal neuron. Scale bar, $50 \mu \mathrm{m}$. $\boldsymbol{D}$, Higher magnification images of the axonal bouton (dotted box in C), the soma of which was marked by an arrow in C. The axon was identified by NF-Himmunofluorescence (blue). Scale bar, $20 \mu \mathrm{m}$. $\boldsymbol{E}-\boldsymbol{G}, \mathrm{Quantitative}$ analysis of colocalization. Ea, Higher magnification image of the FLAG-positive axon (boxed region in C). Other FLAG-negative neurites were eliminated using the binary mask made from NF-H fluorescence image. $\boldsymbol{E b}$, The PDM value was calculated at each pixel and shown as the pixel value at its location. For clarity, the contrast of this PDM image was adjusted where the brightness is saturated at 0.2 . Scale bar, 50 $\mu \mathrm{m} . \boldsymbol{F}$, A scattered plot of green and red pixel intensities against their PDM value at each paired pixel. G, The line profiles of NCKX2 (green) and FLAG-KIF21A (red) fluorescence intensities along two ROI lines (red lines in the left panel). The locations of arrowheads in the right two panels correspond to puncta indicated by arrowheads in Eb. *PDM $>0.1$; **PDM $>0.2$.

points after infection of virus encoding shKIF21A revealed that the expression level of KIF21A was decreased by $73 \%$ of noninfected control levels at $11 \sim 14 \mathrm{~d}$ postinfection (dpi), whereas it was not affected by nontargeting shRNA (NT) control (Fig. 4B). We transfected shKIF21A to hippocampal neurons on
DIV3, and then immunostained them with anti-NCKX $2_{\text {ext }}$ at 15th day after transfection to observe the subcellular distribution of endogenous NCKX2. The KIF21A-depleted cells could be distinguished from untransfected cells by the fluorescence of mRFP contained in the shRNA constructs as an expression marker. Sim- 


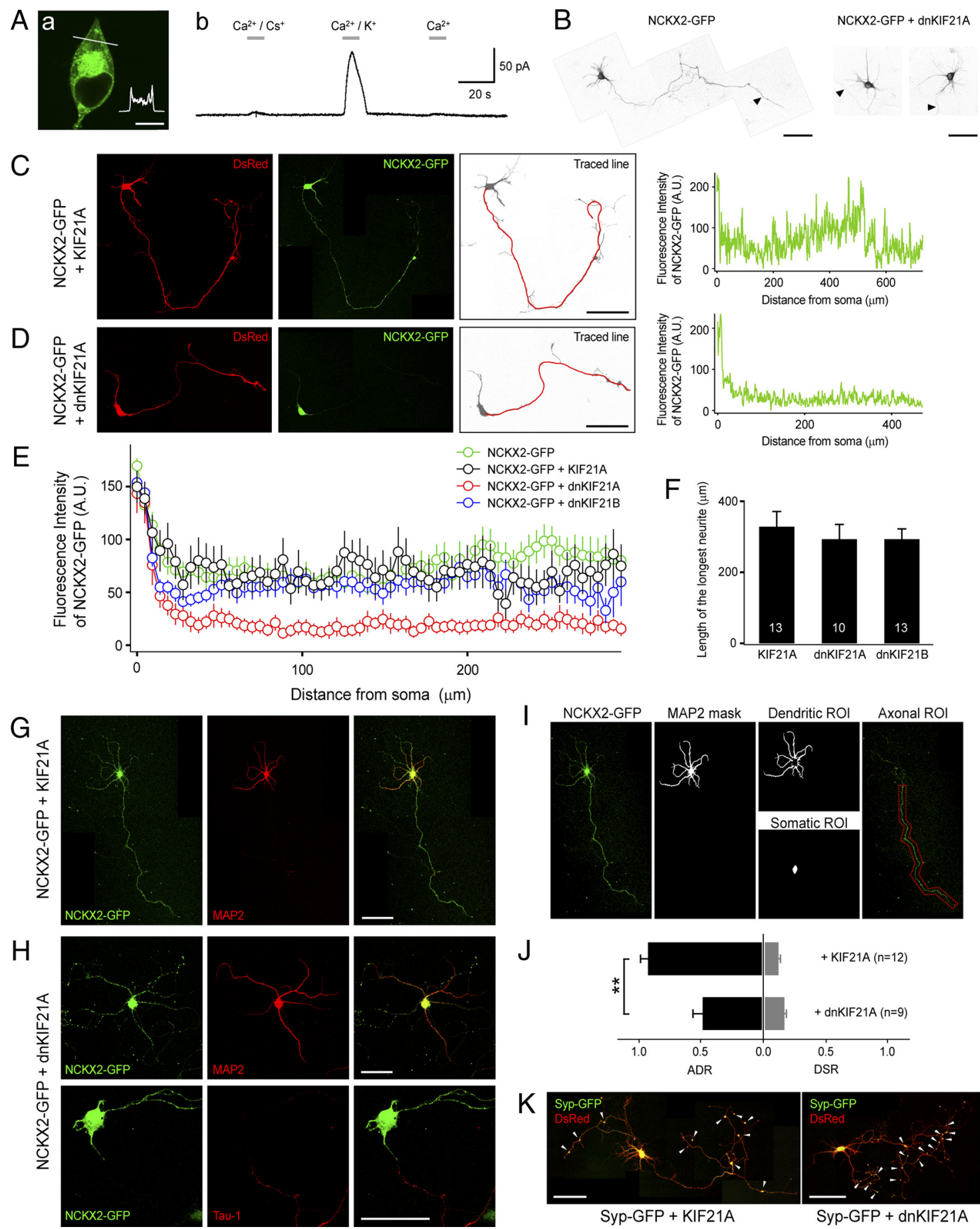

Figure 3. Axonal transport of NCKX2 is inhibited by dnKIF21A. A, Test for functional competence of NCKX2-GFP. Aa, Overexpressed NCKX2-GFP was localized both in the cytoplasm and the plasma membrane in the HEK293 cell. A line profile of GFP fluorescence along the white line is shown in the inset of $\boldsymbol{A} \boldsymbol{a}$. Scale bar, $10 \mu \mathrm{m}$. $\boldsymbol{A} \boldsymbol{b}, \mathrm{A}$ whole-cell current at the holding potential of $0 \mathrm{mV}$ was recorded from a HEK293 cell expressing NCKX2-GFP using high- $\mathrm{Na}^{+}$and high-BAPTA internal pipette solution. Reverse-mode NCKX current could be induced by bath application of $\mathrm{Ca}^{2+}$ plus $\mathrm{K}^{+}$, but not by $\mathrm{Ca}^{2+}$ plus $\mathrm{CS}^{+}$or $\mathrm{Ca}^{2+}$ only. B, Overexpression of NCKX2-GFP alone (left) or coexpression of NCKX2-GFP and dnKIF21A (right) in the hippocampal neurons. GFP fluorescence was inverted into gray color to improve contrast. Scale bar, $100 \mu \mathrm{m}$. The longest neurites were indicated by arrowheads. C, D, Coexpression of NCKX2-GFP together with KIF21A (C) or dnKIF21A (D) in hippocampal neurons. The longest neurite was traced from DsRed fluorescence using NeuronJ, and then fluorescence intensity of NCKX2-GFP was measured along the traced line (rightmost graphs). The traced line is overlaid as a red line on each DsRed image (gray). Scale bar, $100 \mu \mathrm{m}$. E, Mean NCKX2-GFP fluorescence profiles of the longest neurites. Ten points smoothing was performed with all data. In the range of 50-250 $\mu \mathrm{m}$, intensity values for NCKX2-GFP from neurons expressing dnKIF21A (red; $n=11$ ) were significantly lower than those from three different control groups: (1) neurons expressing NCKX2-GFP alone (green; $n=19$ ) at all 43 points, (2) neurons expressing KIF21A (black; $n=10$ ) at 39 points, and (3) neurons expressing dnKIF21B (blue; $n=20)$ at 36 points (mean \pm SEM; $p<0.05)$. No significantly different point was found between NCKX2-GFP-alone and KIF21A and dnKIF21B groups in the same range $(p>0.05)$. $\boldsymbol{F}$, Length of the longest neurites was not significantly different among KIF21A-, dnKIF21A-, and dnKIF21B-expressing groups ( $p>0.5$ ). G, Coexpression of NCKX2-GFP and KIF21A. MAP2 was immunolabeled to distinguish dendrites (red). NCKX2-GFP was localized at both axon and dendrites. Scale bar, $100 \mu \mathrm{m}$. H, Coexpression of dnKIF21A inhibited the transport of NCKX2-GFP to the axon. Dendrites and axon were distinguished by immunolabeling with anti-MAP2 (red; top) and anti-Tau-1 (red; bottom), respectively. Scale bar, $50 \mu \mathrm{m}$. In G and $\boldsymbol{H}$, merged images are shown in the rightmost panels. I, Analysis of ADR of NCKX2-GFP fluorescence. The left two panels show images of NCKX2-GFP fluorescence (the same as $\boldsymbol{G}$ ) and the binary mask of MAP2-positive neurites (white). To make the dendritic (somatic) ROIs, the somatic (dendritic) region was erased from the MAP2 mask (two panels in the third column). The axonal ROI (red dotted polygon in the right panel) was drawn on the NCKX2-GFP image after setting (Figure legend continues) 

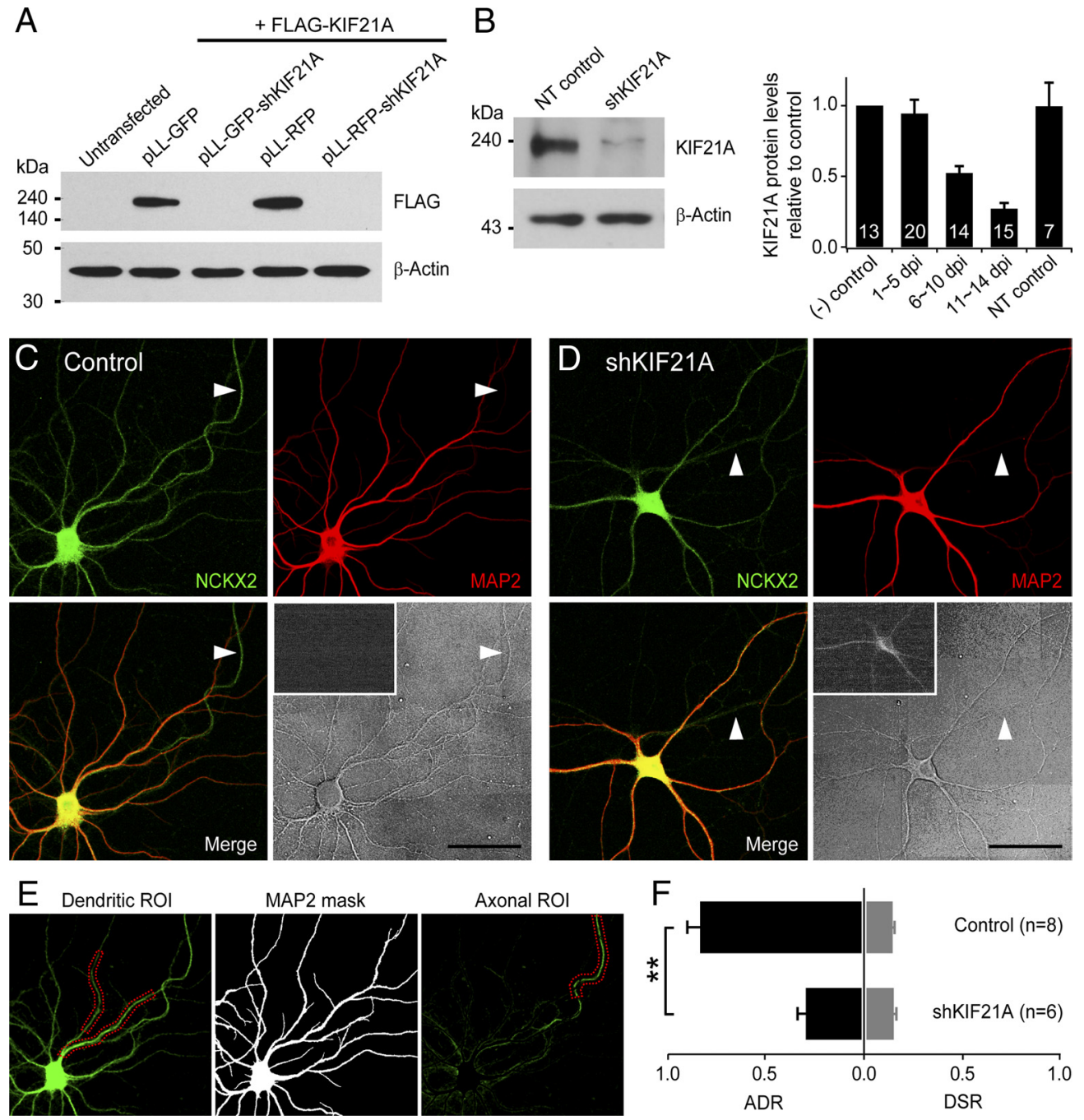

Figure 4. Knockdown of KIF21A inhibits the axonal transport of NCKX2. $\boldsymbol{A}, \boldsymbol{B}$, shRNA-mediated depletion of KIF21A. $\boldsymbol{A}$, KIF21A-targeting shRNA (shKIF21A) was expressed by pLentiLox3.7 plasmids (pLL) encoding EGFP or mRFP (pLL-GFP-shKIF21A or pLL-RFP-shKIF21A). FLAG-KIF21A was cotransfected with shKIF21A or empty pLL vectors into HEK293 cells. shKIF21A completely depleted FLAG-KIF21A, but the empty pLL did not. $B$, Endogenous KIF21A was remarkably depleted in cultured hippocampal neurons infected with lentivirus encoding shKIF21A but not in those with lentivirus encoding nontargeting shRNA (NT control). Time-dependent knockdown of endogenous KIF21A is shown in the right bar graph. The noninfected control is shown in the leftmost bar and the NT control is in the rightmost bar. In $A$ and $B, \beta$-actin was detected as a loading control. $\boldsymbol{C}, \boldsymbol{D}$, The axonal transport of endogenous NCKX2 (green) in the shKIF21A-transfected ( $\boldsymbol{D}$ ) or untransfected control ( $\boldsymbol{C}$ ) neurons. KIF21A-depleted neuron was identified by red fluorescence of mRFP coexpressed with ShRNA (insets on DICimages). Dendrites were identified by MAP2 immunofluorescence (red). Neurites that are MAP2 negative butclearly seen in the DIC images (indicated by arrowheads) were regarded as axons. $E$, Analysis of ADR of endogenous NCKX2. Dendritic ROIs (left; red dotted polygons) were drawn on the endogenous NCKX2 immunofluorescence image (same as in $C$ ). After nullifying pixels that overlap the binary mask of MAP2-positive neurites (middle) from the endogenous NCKX2 image, the axonal ROI was set on the NCKX2 image (right; red dotted polygon). $\boldsymbol{F}$, The mean ADR (black bars) and DSR (gray bars) of endogenous NCKX2 estimated from the untransfected control $(n=8)$ or KIF21A-depleted neurons $(n=6)$. ADR of NCKX2 in the KIF21A-depleted group is significantly lower than that in the control group. ${ }^{* *} p<0.01$. Error bars indicate SEM.

ilar to exogenous NCKX2-GFP (Fig. 3G), endogenous NCKX2 was detected at both the axon and dendrites of the untransfected control (Fig. 4C). In the KIF21A-depleted neuron, however, immunoreactivity of endogenous NCKX2 was reduced in the axonal compartment, but not in the dendritic region (Fig. 4D).

\section{$\leftarrow$}

(Figure legend continued.) the somatodendritic pixels to zero using the MAP2 mask.J, The mean ADR (black bars) and DSR (gray bars) of NCKX2-GFP fluorescence estimated from the neurons cotransfected with KIF21A $(n=12)$ or with dnKIF21A $(n=9)$. ADR of NCKX2-GFP in dnKIF21A group is significantly lower than that in KIF21A group. ${ }^{* *} p<0.01$. Errorbars indicateSEM. $K$, Axonal transport of synaptophysin-GFP was not inhibited by dnKIF21A. To visualize the distribution of synaptophysin, EGFP was fused to the Cterminus of synaptophysin (Syp-GFP; green). Cell morphology was visualized using DsRed fluorescence (red) in the same way as in (and D D. Syp-GFP was well distributed at putative axon terminals (arrowheads), regardless of cotransfection with KIF21A (left) ordnKIF21A (right). Scale bar, $100 \mu \mathrm{m}$.
To quantify the knockdown effect of KIF21A on the axonal transport of endogenous NCKX2, we calculated ADR of NCKX2 immunofluorescence in a similar manner as in Figure $3 I$ (Fig. $4 E$ ). The ADR of endogenous NCKX2 was significantly reduced in the KIF21A-depleted neurons compared with the untransfected control (Fig. 4F; control, $0.832 \pm 0.066, n=8$; shKIF21A, $0.287 \pm 0.043, n=6 ; p<0.01)$. In contrast, the DSR of NCKX2 was not significantly different between control and KIF21Adepleted groups (Fig. 4F; control, $0.135 \pm 0.010, n=8$; shKIF21A, $0.140 \pm 0.014, n=6 ; p>0.1$ ). These results confirm that KIF21A is essential for the axonal transport of NCKX2.

\section{Knockdown of KIF21A alters calcium kinetics at the} axon terminal

Our previous studies revealed that NCKX is the major calcium extrusion machinery at axon terminals but not in the soma (S.-H. Lee 

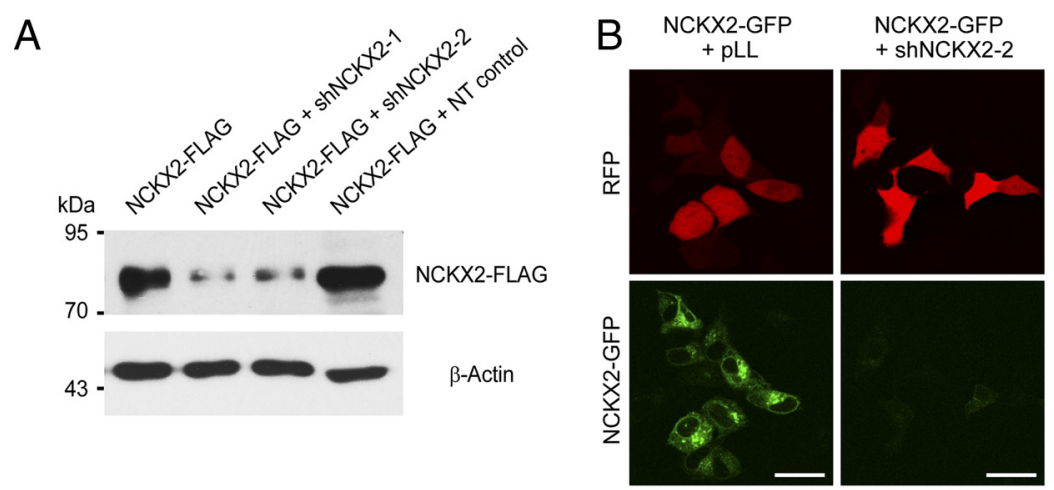

C
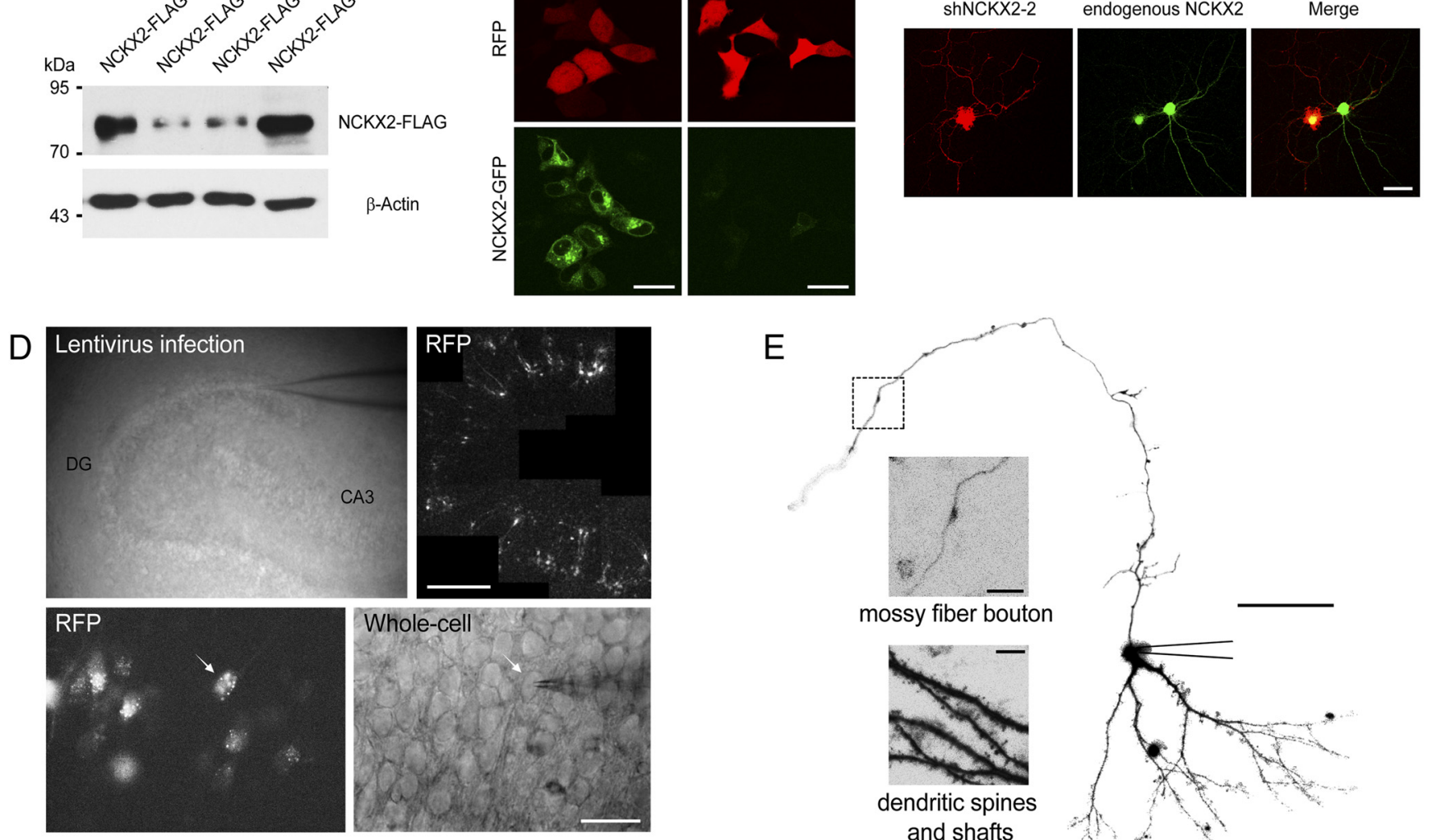

F

G
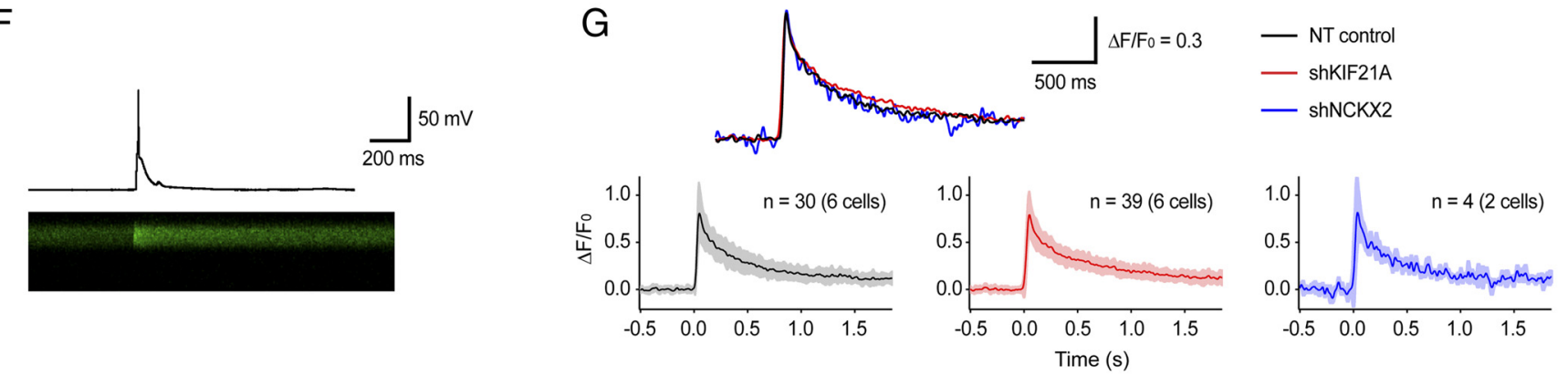
$\mathrm{H} \quad \mathrm{CaT}$ at $33 \mathrm{~Hz} \mathrm{HFS}$
-o- NT control
$\diamond-$ shKIF21A
$\rightarrow$ shNCKX2
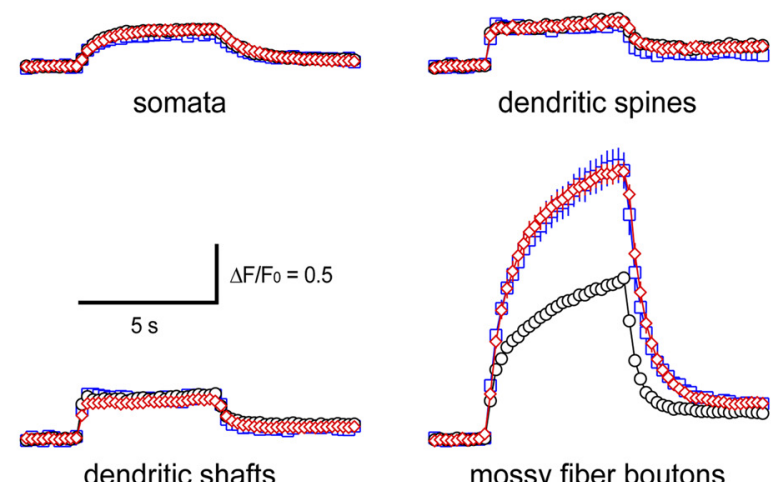

mossy fiber boutons

I CaT at $100 \mathrm{~Hz} \mathrm{HFS}$

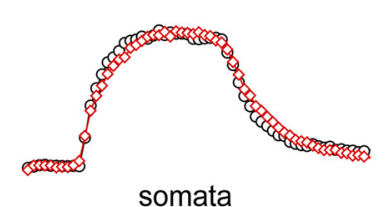

somata

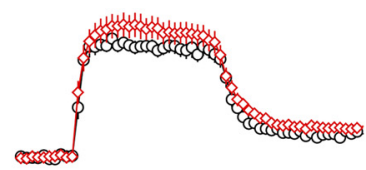

dendritic shafts

Figure 5. Knockdown of KIF21A or NCKX2 enhances CaTs evoked by a high-frequency train of depolarizing pulses (HFS) at MFBs but not in somatodendritic compartments. $\boldsymbol{A}$ - $\boldsymbol{C}$, shRNA-mediated depletion of NCKX2. A, FLAG-tagged NCKX2 (NCKX2-FLAG) was transfected alone or cotransfected with one of two different types of shRNA targeting NCKX2 mRNA (shNCKX2-1 or shNCKX2-2) or nontargeting shRNA (NT control) to HEK293 cells. Both shNCKX2-1 and shNCKX2-2 efficiently depleted NCKX2-FLAG. B, pLentiLox3.7 plasmids (pLL) encoding mRFP alone (red; left) or mRFP plus shNCKX2-2 (red, right) were cotransfected to HEK293 cells with NCKX2-GFP (green). Fluorescence imaging of the cells confirmed that shNCKX2-2 effectively knocked (Figure legend continues) 
et al., 2002, 2009; Kim et al., 2003). We tested whether the depletion of endogenous KIF21A has differential effects on calcium signaling in the soma and axon terminals in hippocampal neurons.

We measured CaTs at mossy fiber boutons (MFBs), en passant boutons of dentate GCs, in hippocampal organotypic slice cultures. On DIV7-DIV8, the slices were infected with lentivirus encoding shKIF21A, shNCKX2 (for its knockdown efficiency, see Fig. 5A-C), or nontargeting shRNA (as a control) by local injection. On 13-17 dpi, we identified lentivirus-infected cells by mRFP fluorescence (Fig. 5D) and loaded the infected GCs with $200 \mu \mathrm{M}$ OGB-5N, a low-affinity $\mathrm{Ca}^{2+}$ indicator dye $\left(K_{\mathrm{D}} \sim 50 \mu \mathrm{M}\right)$, via whole-cell patch pipette (Fig. $5 E$ ). The CaTs were evoked by applying high-frequency stimulation (HFS) (a $5 \mathrm{~s}$ train of AP-like pulses, each comprising a 2 ms depolarization pulse to $0 \mathrm{mV}$ ) delivered at $33 \mathrm{~Hz}$ to the somata of GCs. Because calcium clearance mechanisms undergo developmental change, our analysis was restricted to mature GCs that exhibit low input resistance (<300 M $\Omega$ ) (S.-H. Lee et al., 2009). We traced the mossy fiber under the confocal microscope and identified MFBs by morphological characteristics (D. Lee et al., 2007) (Fig. $5 E$, inset). The plateau $\left[\mathrm{Ca}^{2+}\right]$ level attained by a train of AP-CaTs depends on both calcium clearance and the amplitude of individual AP-CaTs (Regehr et al., 1994). Thus, we confirmed that the mean amplitude of AP-CaTs measured by line scan imaging of OGB-1 fluorescence at the MFBs was not affected by shKIF21A or shNCKX2, ruling out any possible alteration in calcium influx (Fig. $5 F, G$ ).

The mean value for $\left[\mathrm{Ca}^{2+}\right]_{\mathrm{i}}$ increment $\left(\Delta\left[\mathrm{Ca}^{2+}\right]_{\mathrm{i}}\right)$ of HFSinduced CaTs (presented as $\Delta F / F_{0}$ ) at the MFBs of KIF21Adepleted GCs was significantly higher than of control GCs, but was almost the same as that of NCKX2-depleted MFBs [Fig. $5 \mathrm{H}$, peak $\Delta F / F_{0}$ of CaTs in MFBs, $1.302 \pm 0.050$ in the control $(n=$ $64,12$ cells $)$ vs $2.161 \pm 0.079$ in shKIF21A $(n=46,15$ cells; $p<$ $0.01)$ vs $2.171 \pm 0.148$ in shNCKX2 $(n=26,5$ cells; $p<0.01)]$. CaTs in the somata, dendritic shafts, and spines of GCs, however, were not significantly different between control and KIF21A or NCKX2-depleted groups (Fig. 5H, Table 1).

\section{$\leftarrow$}

(Figure legend continued.) down NCKX2-GFP. Scale bar, $30 \mu \mathrm{m}$. C, Endogenous NCKX2 was distinctly depleted by shNCKX2-2 on the 15 th day after transfection. The hippocampal neuron transfected with shNCKX2-2 was identified by coexpressing mRFP fluorescence (red). Endogenous NCKX2 was immunolabeled with anti-NCKX2 $2_{\text {ext }}$ (green) under the permeabilized condition. The persistent green fluorescence confined in the soma of shNCKX2-2-transfected cell is caused by nonspecific staining of nucleus with the secondary antibody because it stained nuclei without a primary antibody under permeabilized conditions (data not shown). Scale bar, 50 $\mu \mathrm{m}$. D, Procedures for viral infection and calcium imaging of hippocampal dentate $\mathrm{GCs}$ in the organotypic cultured slice. The granule cell layer in dentate gyrus of DIV7-DIV8 cultured hippocampal slice was infected with lentivirus encoding shKIF21A, shNCKX2, or NT control by local injection. Lentivirus-infected cells were identified by mRFP fluorescence. Calcium imaging was performed for the RFP-positive dentate GCs loaded with calcium indicator dye via whole-cell patch pipette. Scale bars: top, $200 \mu \mathrm{m}$; bottom, $50 \mu \mathrm{m}$. $\boldsymbol{E}$, AZ-stack projection of laser-scanned images obtained from a dentate GC loaded with $200 \mu \mathrm{M} 0 \mathrm{~GB}-5 \mathrm{~N}$ via a whole-cell patch pipette on the soma. Scale bar, $50 \mu \mathrm{m}$. Insets, Magnified images of the rectangular region of the axon (top), and dendrites (from another cell, bottom). Scale bar: Inset, $5 \mu \mathrm{m}$. $\boldsymbol{F}, \mathbf{G}$, Knockdown of KIF21A did not affect calcium influx evoked by a single AP at MFBs. $\boldsymbol{F}$, Single AP evoked by current injection ( $600 \mathrm{pA}, 12 \mathrm{~ms}$ ) in the soma of a GC (top). A simultaneous line-scanned image recorded from an axon terminal of the $\mathrm{GC}$ loaded with $50 \mu \mathrm{M}$ OGB-1 is shown on the same time axis (bottom). G, Averaged AP-CaTs at MFBs of GCs infected by lentivirus encoding NT control, shKIF21A, or shNCKX2. Traces of AP-CaTs under each condition were smoothed using binomial algorithm (50 passes), and then averaged. The averaged traces are superimposed (top). The trace for each condition is shown with the range of SD (light colored; bottom). $\boldsymbol{H}$, Averaged CaTs in somata, dendritic spines, and shafts and MFBs recorded from GCs infected by lentivirus encoding NT control, shKIF21A, and shNCKX2. CaTs were evoked by HFS ( $33 \mathrm{~Hz}, 5$ s). I, Averaged $\mathrm{CaTs}$ recorded under the conditions similar to $\mathrm{H}$ except HFS at $100 \mathrm{~Hz}$.
Table 1. Mean $\left[\mathrm{Ca}^{2+}\right]_{\mathrm{i}}$ increments induced by HFS of hippocampal dentate granule cells

\begin{tabular}{lllll}
\hline Cell group & Location & $n$ & $\begin{array}{l}\text { No. } \\
\text { of cells }\end{array}$ & $\Delta F / F_{0}$ \\
\hline NT control & Somata & $18(6)$ & $18(6)$ & $0.299 \pm 0.015(0.741 \pm 0.021)$ \\
& Dendritic spines & $34(11)$ & $14(4)$ & $0.357 \pm 0.022(0.577 \pm 0.046)$ \\
& Dendritic shafts & $28(9)$ & $14(5)$ & $0.368 \pm 0.026(0.603 \pm 0.046)$ \\
& MFBs & 64 & 12 & $1.302 \pm 0.050$ \\
shKIF21A & Somata & $19(5)$ & $19(5)$ & $0.300 \pm 0.018(0.724 \pm 0.043)$ \\
& Dendritic spines & $45(11)$ & $13(5)$ & $0.364 \pm 0.029(0.672 \pm 0.047)$ \\
& Dendritic shafts & $35(11)$ & $13(5)$ & $0.306 \pm 0.027(0.673 \pm 0.062)$ \\
shNCKX2 & MFBs & 46 & 15 & $2.161 \pm 0.079^{* *}$ \\
& Somata & 7 & 7 & $0.268 \pm 0.022$ \\
& Dendritic spines & 15 & 4 & $0.329 \pm 0.033$ \\
& Dendritic shafts & 10 & 4 & $0.358 \pm 0.037$ \\
& MFBs & 26 & 5 & $2.171 \pm 0.148^{* *}$
\end{tabular}

$\left[\mathrm{Ca}^{2+}\right]_{\mathrm{i}}$ increments presented as $\Delta F / F_{0}$ of $0 \mathrm{~GB}-5 \mathrm{~N} . \mathrm{Ca}^{2+}$ transients were induced by $\mathrm{HFS}$ at 33 or $100 \mathrm{~Hz}$ (numbers in parentheses). $\Delta F / F_{0}$ values of a $\mathrm{Ca}^{2+}$ transient were read at the end of HFS. NT, Nontargeting. The statistical values are presented as mean $\pm S E M$.

**Statistical significance compared with control value $(p<0.01)$

Previously, we showed that effects of NCKX inhibition on $\mathrm{Ca}^{2+}$ clearance became evident when the $\Delta\left[\mathrm{Ca}^{2+}\right]_{\mathrm{i}}$ was higher than $\sim 0.4 \mu \mathrm{M}$ (Kim et al., 2005). The $\left[\mathrm{Ca}^{2+}\right]_{\mathrm{i}}$ level attained by $\mathrm{HFS}$ at $33 \mathrm{~Hz}$ in the somatodendritic region may not be sufficient to activate NCKX (Fig. 5H), and thus we induced higher CaTs by applying HFS at $100 \mathrm{~Hz}$. Despite the higher plateau $\left[\mathrm{Ca}^{2+}\right]_{\mathrm{i}}$, no difference was found in CaTs between control and KIF21Adepleted groups at somata, dendritic spines, and shafts (Fig. 5I, Table 1), indicating that shKIF21A has no significant effect on the calcium kinetics in the somatodendritic compartment. Therefore, depletion of KIF21A in dentate GCs altered calcium clearance specifically at the MFBs.

\section{Surface expression of NCKX2 is polarized to the axon and its terminals}

It has been demonstrated that the $\mathrm{NaCaX}$ activity in the soma of a dentate GC is independent of intracellular $\mathrm{K}^{+}$, indicative of little NCKX activity (S.-H. Lee et al., 2009). Consistently, knockdown of NCKX2 did not affect the calcium dynamics at the somatodendritic region (Fig. $5 \mathrm{H}$ ). We inquired why not only the axon but also the somatodendritic regions are immunoreactive to NCKX2 (Figs. 2C, $4 C)$. To address this question, we investigated subcellular distribution of NCKX2 expressed on the plasma membrane (surface NCKX2) in cultured hippocampal neurons using live-cell immunocytochemistry with anti-NCKX $2_{\text {ext }}$. In the membranepermeabilized condition, NCKX2-immunoreactive signals were distributed throughout the cell both in the soma and neurites (Figs. 4C, 6Aa). In contrast, the surface NCKX2 signals were colocalized with Tau-1 and a presynaptic protein, synaptophysin (Fig. $6 A b-A d$ ), but not with MAP2 (Fig. 6Ae). To rule out possible effects of endocytosis and exocytosis, we repeated live-cell immunolabeling at $4^{\circ} \mathrm{C}$. The surface expression pattern of $\mathrm{NCKX} 2$ at $4^{\circ} \mathrm{C}$ was similar to that at $36^{\circ} \mathrm{C}$ (Fig. $6 \mathrm{Af}$ ).

We transfected the NCKX2-GFP construct into cultured hippocampal neurons and observed protein localization at 3-10 d after transfection. DsRed was coexpressed to visualize the entire morphology of the neuron. The GFP signal was diffuse throughout the neuron including the axon and dendrites (Fig. $6 \mathrm{Ba}$ ). Livecell immunostaining with anti-GFP antibody, however, revealed that the immunoreactivity of surface GFP was not colocalized with MAP2, but it was detected on the MAP2-negative neurites (Fig. $6 \mathrm{Bb}$ ). Thus, surface NCKX2-GFP exists along the axon, similar to endogenous NCKX2. These results indicate that surface expression 


\section{A Endogenous NCKX2}

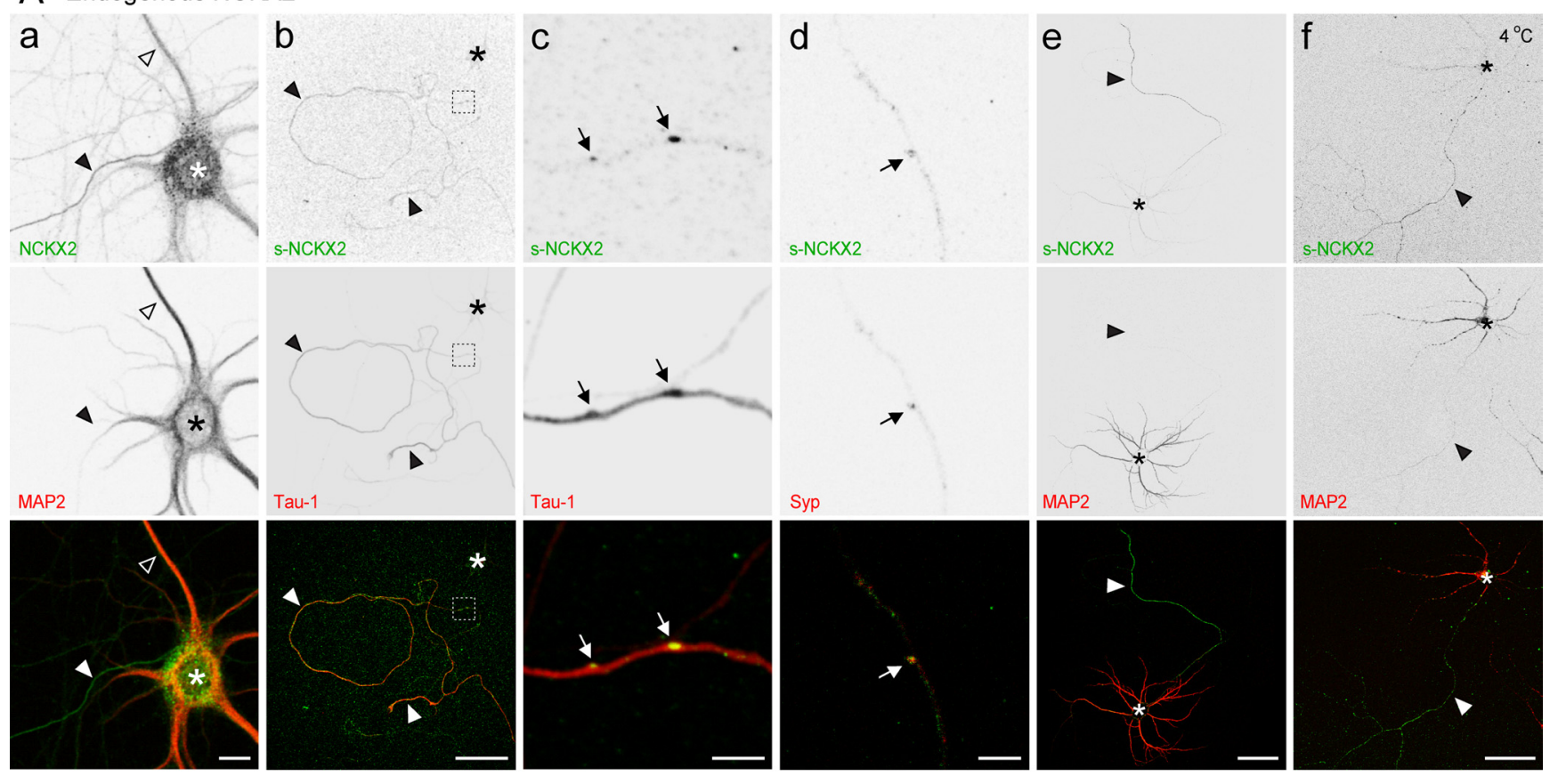

\section{B Exogenous NCKX2-GFP}

a
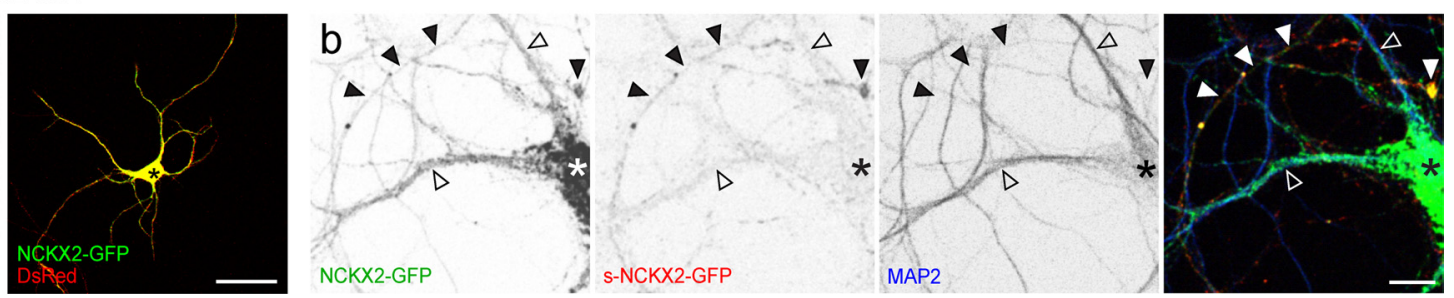

Figure 6. Immunocytochemical localization of endogenous ( $\boldsymbol{A}$ ) or exogenous (B) NCKX2 in cultured hippocampal neurons. $A a$, DIV23 hippocampal neurons were stained for endogenous NCKX2 (green) and dendritic marker MAP2 (red). $\boldsymbol{A} \boldsymbol{b}$-Ae, Surface NCKX2 (s-NCKX2) was immunolabeled by incubating live cells with anti-NCKX2 ext (green) at $36^{\circ} \mathrm{C}$, and then cells were fixed, permeabilized, and immunostained with antibodies against axonal marker Tau-1 (red; $\boldsymbol{A b}, \boldsymbol{A c}$ ) or presynaptic marker synaptophysin (red; $\boldsymbol{A d}$ ) or dendritic maker MAP2 (red; $\boldsymbol{A e}$ ). Higher magnification image of the dashed box in $\boldsymbol{A} \boldsymbol{b}$ is shown in $\boldsymbol{A c}$. The sites where endogenous s-NCKX2 was colocalized with Tau-1 (Ac) or synaptophysin (Ad) are marked with arrows. $\boldsymbol{A}$, Endogenous s-NCKX2 (green) was immunolabeled by the same manner as in $\boldsymbol{A e}$ except incubating live cells with anti-NCKX $2_{\text {ext }}$ at $4^{\circ}$ C before fixation. Scale bars: $\boldsymbol{A a}, 10 \mu \mathrm{m} ; \boldsymbol{A b}, \boldsymbol{A e}, \boldsymbol{A f}, 50 \mu \mathrm{m} ; \boldsymbol{A c}, \boldsymbol{A d}, 5 \mu \mathrm{m}$. Ba , A DIV7 hippocampal neuron transfected with NCKX2-GFP and DsRed. Scale bar, $50 \mu \mathrm{m}$. Bb , A DIV13 hippocampal neuron transfected with NCKX2-GFP (green). Surface NCKX2-GFP was visualized by live-cell immunolabeling with antibody against GFP (s-NCKX2-GFP; red), and then stained for MAP2 (blue). Scale bar, $10 \mu \mathrm{m}$. The open and solid arrowheads indicate dendrites and axons, respectively. The asterisks show location of somata.

of not only endogenous but also exogenous NCKX2 is highly polarized to the axon and its terminals, and support the results of the functional studies.

Endocytosis restricts the surface expression of NCKX2 on the somatodendritic compartment

Under permeabilized conditions, NCKX2 immunoreactivity was positive not only in the axon but also in the somatodendritic compartment where we could not observe NCKX activity (Kim et al., 2003; S.-H. Lee et al., 2009). The discrepancy between morphological and functional results could be reconciled by the differential surface expression of NCKX2 between the axonal and somatodendritic compartments (Fig. 6). The differential surface and cytosolic distribution of NCKX2 in the dendritic compartment implies that the axonal targeting of NCKX2 is regulated by the selective retention model (Sampo et al., 2003). According to this model, we hypothesized that the cytoplasmic NCKX2 may be transported to the dendrite and then eliminated from the dendritic surface. To test this hypothesis, we investigated whether inhibition of endocytosis has an effect on the somatodendritic surface expression of NCKX2. When the NCKX2-GFP- expressing hippocampal neurons were treated with dynasore (40 $\mu \mathrm{M}$, for $4 \mathrm{~h}$ ), a cell-permeable dynamin inhibitor, they exhibited greatly increased surface expression of NCKX2-GFP in the somatodendritic region, compared with the neurons treated with vehicle $(0.1 \%$ DMSO) (Fig. $7 A)$. To quantify the polarization of surface expression, we calculated the ADR of surface NCKX2GFP using the binary mask of MAP2-positive and -negative neurites of a cell created from the fluorescence image of NCKX2-GFP (Fig. 7C) (for details, see Materials and Methods). The ADR of surface NCKX2-GFP was significantly lower in the dynasoretreated neurons than in the vehicle-treated control (Fig. 7D; control, $3.436 \pm 1.120, n=6$; dynasore-treated group, $0.831 \pm$ $0.127, n=7$; $p<0.05)$. Similar to exogenous NCKX2-GFP, the endogenous NCKX2 was notably increased on the somatodendritic surface by dynasore (Fig. 7E, F; control, $14.62 \pm 1.22, n=$ 7 ; dynasore, $24.31 \pm 3.56, n=9 ; p<0.01$ ). As an alternative way to block clathrin-mediated endocytosis, we used a dominantnegative mutant of dynamin-1, which was created by replacing the lysine residue with alanine at the amino acid position 44 (DYN1-K44A) (Damke et al., 1994). NCKX2-FLAG was coexpressed together with wild-type dynamin-1 (DYN1-WT) or 

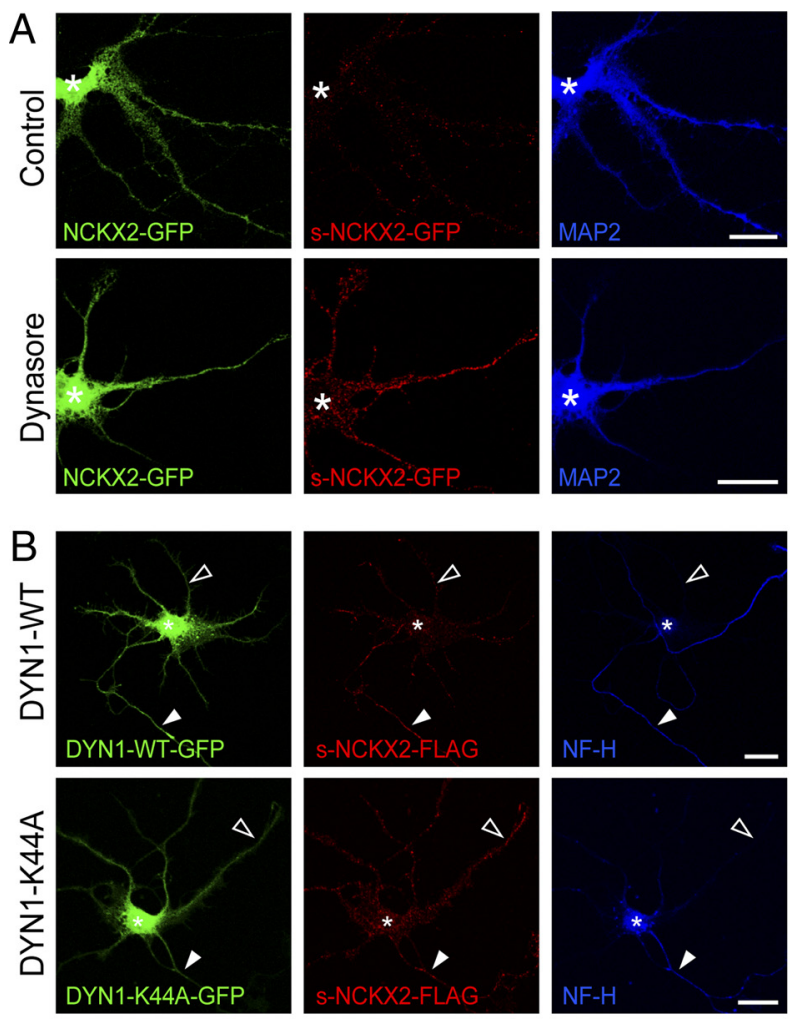

C
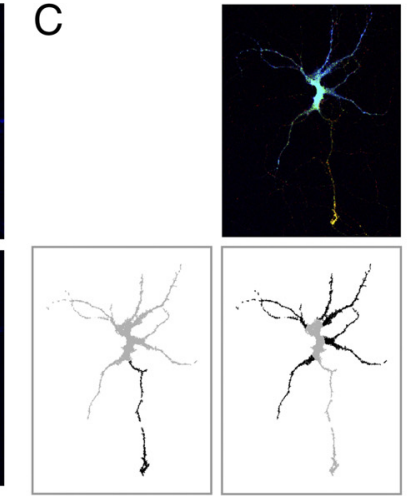

D

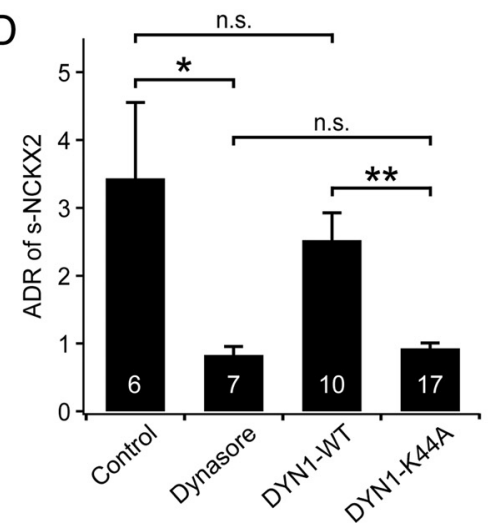

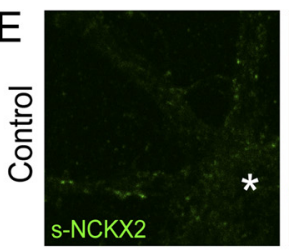
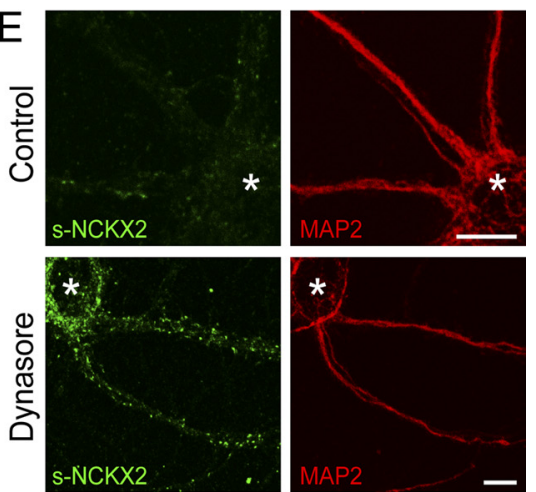

$\mathrm{F}$

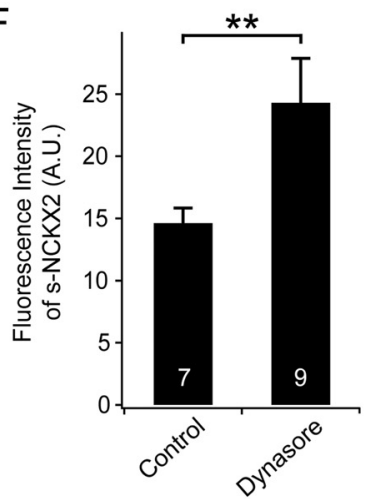

Figure 7. Inhibition of endocytosis enhances the surface expression of NCKX2 in the somatodendritic region. $A$, Hippocampal neurons expressing NCKX2-GFP were treated with $0.1 \%$ DMSO (control; top) or $40 \mu \mathrm{m}$ dynasore (bottom) for $4 \mathrm{~h}$. Surface NCKX2-GFP (s-NCKX2-GFP) was visualized with anti-GFP (red). Dendrites were immunostained with anti-MAP2 (blue). Scale bar, $20 \mu \mathrm{m}$. B, Wild-type dynamin-1 (DYN1-WT) or dominant-negative mutant dynamin-1 (DYN1-K44A) was cotransfected with NCKX2-FLAG to DIV6 hippocampal neurons. Both DYN1-WT and DYN1-K44A were tagged with EGFP on their $\mathrm{N}$-terminals, and transfected cells were identified by green fluorescence (left). Surface NCKX2-FLAG (s-NCKX2-FLAG, red) was detected by anti-FLAG. Axons were immunostained with anti-NF-H (blue). The open and solid arrowheads indicate dendrites and axons, respectively. Scale bar, $20 \mu \mathrm{m}$. C, Analysis of the ADR of s-NCKX2-GFP. Top, A merged image of three colors from the control neuron shown in $A$. Note only the axonal compartment exhibits the colocalization (yellow) of s-NCKX2-GFP (red) and NCKX-GFP (green). Bottom panels, Each panel shows a binary mask image of the same cell created from NCKX2-GFP fluorescence (gray). The MAP2-negative axonal ROI (left) or MAP2-positive dendritic ROls (right) are overlaid in black. D, Summary for the mean ADR ofs-NCKX2-GFP in A ors-NCKX2-FLAG in B. The ADRestimated from the neurons treated with dynasore and those transfected with DYN1-K44A are significantly lower than that from the control neurons. $\boldsymbol{E}$, Surface expression of endogenous NCKX2 (s-NCKX2) was immunostained with anti-NCKX2 $2_{\text {ext }}$ (green). Dendrites were immunostained with anti-MAP2 (red). Scale bar, $10 \mu \mathrm{m}$. $\boldsymbol{F}$, The mean fluorescence intensity of endogenous s-NCKX2 on MAP2-positive neurites of the control or dynasore-treated groups. Surface NCKX2 (endogenous or EGFP-tagged or FLAG-tagged) was detected by means of live-cell immunolabeling $(A, B, E)$. The asterisks in the micrographs indicate the locations of somata. ${ }^{* *} p<0.01 ;{ }^{*} p<0.05 ;$;.S., not significant. Error bars indicate SEM.

DYN1-K44A. Similar to dynasore, overexpression of DYN1K44A increased the surface expression level of NCKX2-FLAG in the somatodendritic compartment, whereas DYN1-WT had little effect (Fig. 7B, D; DYN1-WT, $2.52 \pm 0.40, n=10$; DYN1-K44A, $0.93 \pm 0.08, n=17 ; p<0.01)$. These results demonstrate that preferential endocytosis of NCKX2 from the somatodendritic surface together with KIF21A-mediated axonal transport of NCKX2 underlies the polarized axonal targeting of NCKX2.

Inhibition of endocytosis allows the NCKX activity to emerge in the dendritic region of GCs

To confirm the hypothesis that endocytosis suppresses the surface expression of NCKX2 in the somatodendritic region, we tested whether pretreatment of GCs with dynasore enhances the NCKX activity in their proximal dendrites. To estimate $\mathrm{Ca}^{2+}$ clearance, we evoked a CaT in a GC with a short depolarizing pulse under eight different conditions: normal or low extracellular $\left[\mathrm{Na}^{+}\right]\left(\left[\mathrm{Na}^{+}\right]_{\text {ext }}\right) ; \mathrm{K}^{+}$- or $\mathrm{TMA}^{+}$-based pipette solution $\left(\mathrm{K}_{\mathrm{i}}\right.$ or $\mathrm{TMA}_{\mathrm{i}}$, respectively); pretreatment with dynasore or DMSO as vehicle. Averaged CaTs in each condition are compared in Figure $8 \mathrm{~A}$. Pretreatment with dynasore $(40 \mu \mathrm{M}$, for an hour) accelerated the $\mathrm{Ca}^{2+}$ decay rate under normal $\left[\mathrm{Na}^{+}\right]_{\text {ext }}$ and $\mathrm{K}_{\mathrm{i}}$ conditions but not under low $\left[\mathrm{Na}^{+}\right]_{\text {ext }}$ or $\mathrm{TMA}_{\mathrm{i}}$ conditions (Fig. $8 \mathrm{~A}$ ). Averaged traces for CaTs in normal and low $\left[\mathrm{Na}^{+}\right]_{\text {ext }}$ are compared in each panel of Figure $8 B$ under four different conditions as indicated. We fitted a biexponential function to the decay phase of an individual $\mathrm{CaT}$ and regarded the weighted average of rate constants $\left(r_{\mathrm{w}}\right)$ of the fitted function as a parameter for $\mathrm{Ca}^{2+}$ clearance (S.-H. Lee et al., 2009). We estimated the NaCaX activity in a GC as a difference between the $r_{\mathrm{w}}$ value measured in the normal $\left[\mathrm{Na}^{+}\right]_{\text {ext }}$ and that in the low $\left[\mathrm{Na}^{+}\right]_{\text {ext }}$ (Fig. $8 \mathrm{C}$ ). Consistent with our previous report (S.-H. Lee et al., 2009), the dendritic $\mathrm{NaCaX}$ activity was little altered by $\mathrm{TMA}^{+}$-based pipette solution, indicating that most $\mathrm{NaCaX}$ activity can be attributed to NCX rather than NCKX (Fig. 8Cc; K $, 1.58 \pm 0.26 / \mathrm{s}, n=7$; TMA, $1.22 \pm$ $0.16 / s, n=5 ; p>0.1)$. In contrast, pretreatment of the GCs with dynasore greatly enhanced the $\mathrm{NaCaX}$ activity under the $\mathrm{K}_{\mathrm{i}}$ condition (Fig. $8 C c ; 3.00 \pm 0.21 / s, n=5 ; p<0.01$ ), but not under the $\mathrm{TMA}_{\mathrm{i}}$ condition in which the $\mathrm{NaCaX}$ activity was rather decreased from unknown reasons (Fig. $8 C c ; 0.78 \pm 0.12 / \mathrm{s}, n=6$; $p<0.05)$. These results indicate that dynasore enhances the dendritic $\mathrm{NaCaX}$ activity and that the effect of dynasore requires intracellular $\mathrm{K}^{+}$. Therefore, these observations strongly suggest that NCKX rather than NCX is responsible for the NaCaX activity enhanced by dynasore in the proximal dendrite. We conclude 

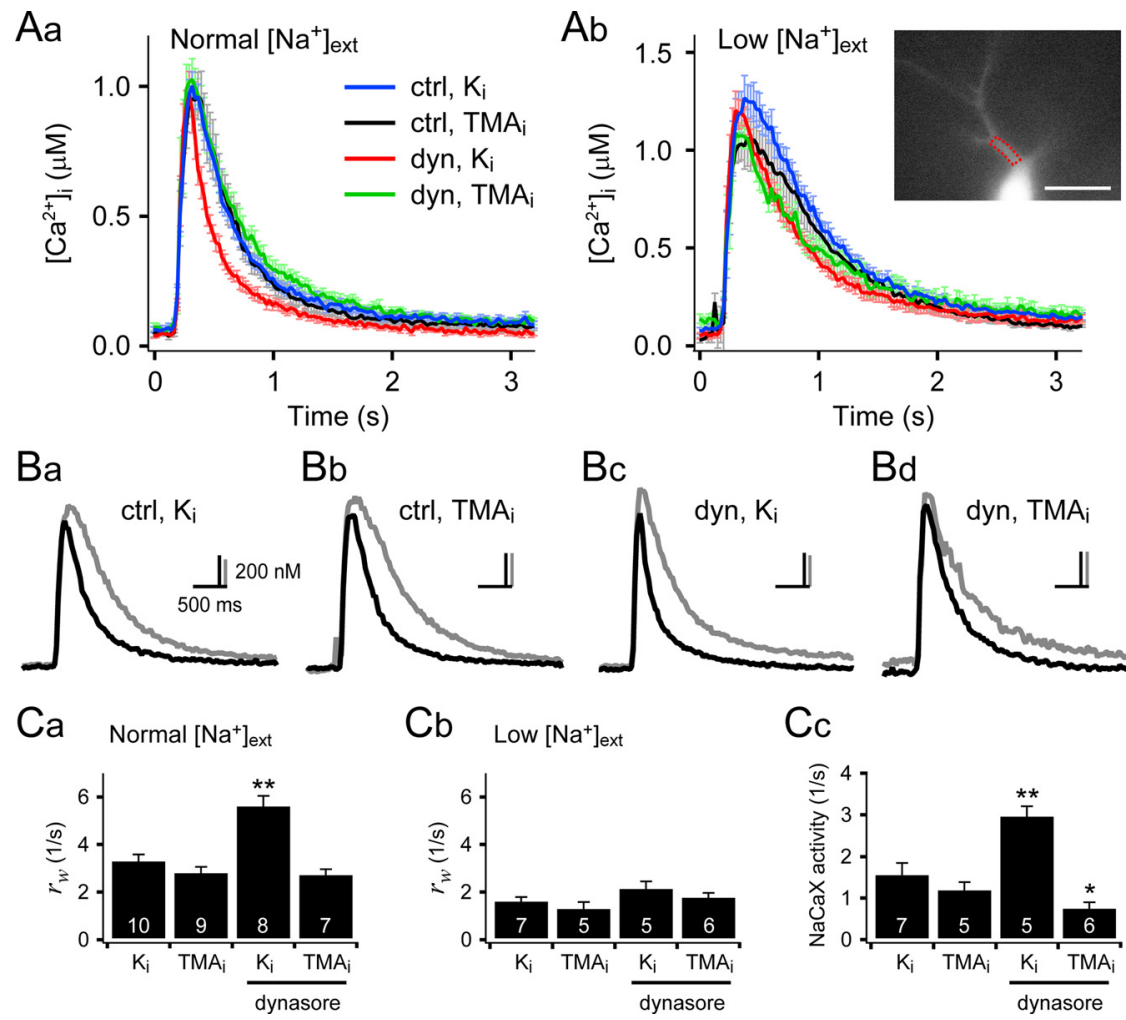

$\mathrm{Bb}$

$\mathrm{Bc}$

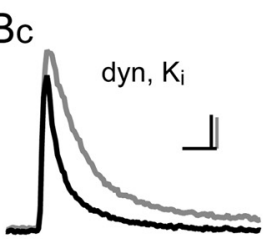

$\mathrm{Bd}$

$\mathrm{Cc}$

Figure 8. Dynasore enhances the NCKX activity in the proximal dendrite of hippocampal dentate $\mathrm{GCS}$. $\boldsymbol{A}$, Averaged traces of $\mathrm{CaTs}$ evoked by a short depolarizing pulse (from -70 to $0 \mathrm{mV}$ for $50 \sim 100 \mathrm{~ms}$ ) under normal $(A a)$ or low $(A b)$ extracelluar $\left[\mathrm{Na}^{+}\right.$] $\left(\left[\mathrm{Na}^{+}\right]_{\text {ext }}\right)$ conditions. Each panel shows four overlaid averaged traces under different conditions $\left(\mathrm{K}_{\mathrm{i}}\right.$ or TMA $\mathrm{TM}_{\mathrm{i}}, \mathrm{K}^{+}$- or TMA ${ }^{+}$-based pipette solution; ctrl or dyn, pretreatment with vehicle or $40 \mu \mathrm{m}$ dynasore for $1 \mathrm{~h}$, respectively). Note that pretreatment with dynasore accelerated the $\mathrm{Ca}^{2+}$ decay rate only under $\mathrm{K}^{+}$-based internal and normal $\left[\mathrm{Na}^{+}\right]_{\text {ext }}$ conditions (red lines). Error bars shown in light colors represent SEM. Inset, A fura-4F fluorescence image of a GC. A typical proximal dendritic ROl is shown as a red dotted box. Scale bar, $20 \mu \mathrm{m}$. B, Slowdown of $\mathrm{Ca}^{2+}$ decay rate in the low $\left[\mathrm{Na}^{+}\right]_{\text {ext }}$ condition. Each panel shows overlaid averaged $\mathrm{CaT}$ recorded under the same condition except $\left[\mathrm{Na}^{+}\right]_{\text {ext }}$ (black, normal $\left[\mathrm{Na}^{+}\right]_{\text {ext }}$ gray, low $\left.\left[\mathrm{Na}^{+}\right]_{\text {ext }}\right)$. C, Summary for $\mathrm{Ca}^{2+}$ clearance and $\mathrm{NaCaX}$ activity. $\mathrm{Ca}^{2+}$ clearance was quantified as a weighted average of rate constants $\left(r_{\mathrm{w}}\right)$ estimated from the biexponential fit to the decay phase of individual $\mathrm{CaTs}(\boldsymbol{C a}, \boldsymbol{C b})$. The difference in the $r_{\mathrm{w}}$ values between normal and low $\left[\mathrm{Na}^{+}\right]_{\mathrm{ext}}$ conditions in the same cell was regarded as the $\mathrm{NaCaX}$ activity $(\mathbf{C C})$. Shown are mean $\pm \mathrm{SEM} .{ }^{* *} p<0.01 ;{ }^{*} p<0.05$.

that the surface expression of NCKX is restricted by endocytosis in the somatodendritic region.

\section{Discussion}

NCKX activity was first discovered at the retinal photoreceptor, and now it is recognized that isoforms of NCKX are widely distributed in the brain (for review, see Lytton, 2007). In the brain, NCKX activity was first observed at neurohypophysial axon terminals, although no NCKX activity was observed in the somata of the same neurons, located in the supraoptic nucleus (SON) of the hypothalamus (S.-H. Lee et al., 2002; Kim et al., 2003). Several lines of evidence support the idea that NCKX2 is responsible for the NCKX activity at these axon terminals. First, the affinity for intracellular $\mathrm{K}^{+}$of the NCKX activity at the neurohypophysial axon terminal was distinctly low and compatible to that of NCKX2 (S.-H. Lee et al., 2002; Visser et al., 2007). Second, transcripts of NCKX2, revealed by in situ hybridization analysis, were more prominent than other isoforms in magnocellular neurons of the SON (S.-H. Lee et al., 2002). Third, the NCKX activity at the glutamatergic presynaptic terminal in medial nucleus of the trapezoid body, a calyx of Held, was enhanced by phorbol ester, an activator of PKC, and NCKX2 is the only isoform modulated by PKC (Kim et al., 2005; J. Y. Lee et al., 2006). These results imply

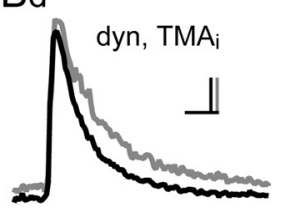

that NCKX2 is the most plausible isoform responsible for calcium clearance at axon terminals in central neurons.

In the present study, we identified a novel interaction between NCKX2 and KIF21A, and demonstrated that KIF21A is essential for the axonal transport of NCKX2 (Figs. 3, 4). Recently, it has been proposed that the AIS, comprised of F-actin, spectrin, and ankyrin-G, functions as a checkpoint to axonal protein transport (Song et al., 2009). The AIS not only initiates action potentials but also functions as a selective filter that regulates axonal transport (Grubb and Burrone, 2010). The AIS is not just a physical barrier that screens a molecule by its size, but a highly flexible structure that is capable of selecting specific molecular components. For example, the axonal entry of $70 \mathrm{kDa}$ dextrans was prevented by AIS (Song et al., 2009), but the large $360 \mathrm{kDa} \mathrm{K} 3.1 \mathrm{~b}$ tetramer that interacts with ankyrin-G could pass through the AIS (Xu et al., 2010). Therefore, axonal transport of a molecule requires not only a molecular motor (kinesin or myosin) but also interaction of motor-cargo complex with AIS components, which allows the complex to pass through the AIS. Recently, it has been reported that the third and fourth coiledcoil domains of the KIF21A stalk can interact with the ankyrin-repeat domain (Kakinuma and Kiyama, 2009), implying that the KIF21A (motor)-NCKX2 (cargo) complex is transported to the axon by interacting with ankyrin-G of AIS.

We showed that overexpression of dnKIF21A or depletion of KIF21A did not alter the DSR of NCKX2 (Figs. 3J, 4F). These findings, however, cannot be direct evidence for little role of KIF21A in the dendritic transport of NCKX2 because NCKX2 molecules in dendritic endoplasmic reticulum may be responsible for the dendritic immunoreactivity. Furthermore, the colocalization of KIF21A with NCKX2 in dendrites implies a possible role of KIF21A in the dendritic transport (Fig. 2C). Thus, it remains to be elucidated whether KIF21A plays an essential role in dendritic transport or not.

The consistent effects of dominant-negative KIF21A and knockdown of KIF21A expression on NCKX2 delivery to the axon indicate that KIF21A mediates the axonal transport of NCKX2 in hippocampal neurons. Mutations in KIF21A cause congenital fibrosis of extraocular muscle type I (CFEOM1), which is a disorder of the superior oculomotor nerve (Yamada et al., 2003). The prevalent hypothesis for the pathogenesis of CFEOM1 is aberrant axonal routing and resultant abnormal development of the oculomotor axis (Engle et al., 1997; Heidary et al., 2008). Given that calcium plays a crucial role in axon guidance (Gomez and Zheng, 2006), a defect in axonal transport of NCKX2 might be the direct cause of CFEOM1. Therefore, we studied whether functional inactivation of NCKX2 brings about pathological changes similar to CFEOM1 using NCKX2 knockout mice (Li et al., 2006). We found, however, no obvious differ- 
ence in the morphology of $\alpha$ motor neurons in the oculomotor nucleus between wild-type and knock-out (data not shown), implying that CFEOM1 is not caused directly by a functional defect in NCKX2. Of course, one should be cautious in extending the observations from mice to humans because mice have no subdivision in the oculomotor nucleus observed in the human oculomotor complex.

The depletion of KIF21A caused calcium dysregulation at axon terminals, whereas the somatodendritic compartment was spared (Fig. 5). This finding can be explained in light of our results that surface expression of NCKX2 in the plasma membrane is confined to the axon and its terminals (Fig. 6) and that KIF21A is required for axonal transport of NCKX2 (Figs. 3, 4). Furthermore, we observed that the surface expression of NCKX2 did not conform to the subcellular distribution of total (surface plus cytosolic) NCKX2 in the neuron (Fig. 6). Similar to NCKX2, several axonally targeted proteins such as $\mathrm{Na}_{\mathrm{v}} 1.2$ (Garrido et al., 2001), VAMP2 (Sampo et al., 2003), $\beta 1$-integrin (Gut et al., 1998), and Caspr2 (Bel et al., 2009) have been found to exhibit differential surface and cytoplasmic distribution. The somatodendritic endocytosis (selective retention mechanism) has been proposed as a mechanism for the axonspecific surface expression of these proteins. The present study demonstrated that dynamin-dependent endocytosis of NCKX2 occurs preferentially in the somatodendritic compartment and contributes to the polarized axonal surface expression of NCKX2 (Fig. 7). Therefore, we conclude that the polarized axonal targeting of NCKX2 is achieved by KIF21A-mediated axonal transport in concert with somatodendritic endocytosis of NCKX2 in hippocampal neurons.

Our previous analyses of $\mathrm{Ca}^{2+}$ clearance in the somata revealed that the somatic $\mathrm{NaCaX}$ activity is independent of intracellular $\mathrm{K}^{+}$in the hippocampal GC and the supraoptic magnocellular neuron, indicating lack of NCKX in their surface of somata (Kim et al., 2003; S.-H. Lee et al., 2009). Nevertheless, NCKX activity in the soma has been reported by other studies in the hippocampal CA1 pyramidal and cortical neurons (Kiedrowski, 2004; Cuomo et al., 2008). The discrepancy can result from different types or developmental ages of the central neurons studied (S.-H. Lee et al., 2007). More importantly, it remains to be elucidated whether neurons in the CNS are equipped with a mechanism regulating the surface expression of NCKX2 in the somatodendritic compartment. In light of the present study, somatodendritic endocytosis of NCKX2, which is essential for maintaining the polarized axonal targeting, is one of the potential regulation points for the somatodendritic surface expression of NCKX2. Clathrin-mediated endocytosis is initiated by recruitment of AP-2, an adaptor protein. There are two well known canonical endocytosis motifs that interact with AP-2. One is a dileucine motif (DxxLL; $x=$ any amino acid) recognized by the $\beta 2$ subunit of AP-2 (Rapoport et al., 1998), and the other is a tyrosine motif (Yxx $\phi ; \phi=$ hydrophobic amino acid) recognized by the $\mu 2$ subunit of AP-2 (Ohno et al., 1995). We found that the intracellular loop region of NCKX2 has two tyrosine motifs $\left({ }^{365} \mathrm{YGKL}^{368}\right.$ and $\left.{ }^{371} \mathrm{YDTM}^{374}\right)$. Intriguingly, these two tyrosine motifs are located in the alternative splicing region of NCKX2 (amino acids 361-377) (Tsoi et al., 1998). It remains to be clarified whether the interaction between NCKX2 and $\mu 2$ subunit of AP-2 is essential for endocytosis of the somatodendritic NCKX2. These endocytosis motifs can be removed by alternative splicing, raising a possibility that such a spliced isoform of NCKX2 may be expressed in the surface of the somatodendritic compartment. Therefore, alternative splicing may be one of potential regulation mechanisms for the polarized targeting of NCKX2.
The present study demonstrated that the polarized axonal targeting of NCKX2 arises through the concerted contribution of two mechanisms: KIF21A-mediated axonal transport and preferential somatodendritic endocytosis. This finding implies that there is room for diverse subcellular surface expression of NCKX2 depending on the regulation of the two mechanisms. The regulatory mechanism for the subcellular surface expression of NCKX2 could be essential for understanding the cell-specific role of NCKX2 in $\mathrm{Ca}^{2+}$ homeostasis, which is relevant to synaptic plasticity and alleviation of ischemic brain injury (Li et al., 2006; Cuomo et al., 2008).

\section{References}

Arnold DB (2007) Polarized targeting of ion channels in neurons. Pflugers Arch 453:763-769.

Bel C, Oguievetskaia K, Pitaval C, Goutebroze L, Faivre-Sarrailh C (2009) Axonal targeting of Caspr2 in hippocampal neurons via selective somatodendritic endocytosis. J Cell Sci 122:3403-3413.

Braam SR, Denning C, Matsa E, Young LE, Passier R, Mummery CL (2008) Feeder-free culture of human embryonic stem cells in conditioned medium for efficient genetic modification. Nat Protoc 3:1435-1443.

Cai X, Zhang K, Lytton J (2002) A novel topology and redox regulation of the rat brain $\mathrm{K}^{+}$-dependent $\mathrm{Na}^{+} / \mathrm{Ca}^{2+}$ exchanger, NCKX2. J Biol Chem 277:48923-48930.

Cuomo O, Gala R, Pignataro G, Boscia F, Secondo A, Scorziello A, Pannaccione A, Viggiano D, Adornetto A, Molinaro P, Li XF, Lytton J, Di Renzo G, Annunziato L (2008) A critical role for the potassium-dependent sodium-calcium exchanger NCKX2 in protection against focal ischemic brain damage. J Neurosci 28:2053-2063.

Damke H, Baba T, Warnock DE, Schmid SL (1994) Induction of mutant dynamin specifically blocks endocytic coated vesicle formation. J Cell Biol 127:915-934.

Davis HE, Rosinski M, Morgan JR, Yarmush ML (2004) Charged polymers modulate retrovirus transduction via membrane charge neutralization and virus aggregation. Biophys J 86:1234-1242.

De Simoni A, Yu LM (2006) Preparation of organotypic hippocampal slice cultures: interface method. Nat Protoc 1:1439-1445.

Dotti CG, Sullivan CA, Banker GA (1988) The establishment of polarity by hippocampal neurons in culture. J Neurosci 8:1454-1468.

Engle EC, Goumnerov BC, McKeown CA, Schatz M, Johns DR, Porter JD, Beggs AH (1997) Oculomotor nerve and muscle abnormalities in congenital fibrosis of the extraocular muscles. Ann Neurol 41:314-325.

Garrido JJ, Fernandes F, Giraud P, Mouret I, Pasqualini E, Fache MP, Jullien F, Dargent B (2001) Identification of an axonal determinant in the C-terminus of the sodium channel $\mathrm{Na}_{\mathrm{v}}$ 1.2. EMBO J 20:5950-5961.

Gomez TM, Zheng JQ (2006) The molecular basis for calcium-dependent axon pathfinding. Nat Rev Neurosci 7:115-125.

Grubb MS, Burrone J (2010) Building and maintaining the axon initial segment. Curr Opin Neurobiol 20:481-488.

Gut A, Balda MS, Matter K (1998) The cytoplasmic domains of a $\beta 1$ integrin mediate polarization in Madin-Darby canine kidney cells by selective basolateral stabilization. J Biol Chem 273:29381-29388.

Heidary G, Engle EC, Hunter DG (2008) Congenital fibrosis of the extraocular muscles. Semin Ophthalmol 23:3-8.

Hioki H, Kuramoto E, Konno M, Kameda H, Takahashi Y, Nakano T, Nakamura KC, Kaneko T (2009) High-level transgene expression in neurons by lentivirus with Tet-Off system. Neurosci Res 63:149-154.

Hirokawa N, Takemura R (2005) Molecular motors and mechanisms of directional transport in neurons. Nat Rev Neurosci 6:201-214.

Hirokawa N, Niwa S, Tanaka Y (2010) Molecular motors in neurons: transport mechanisms and roles in brain function, development, and disease. Neuron 68:610-638.

Hong YH, Kim JY, Lee JH, Chae HG, Jang SS, Jeon JH, Kim CH, Kim J, Kim SJ (2009) Agonist-induced internalization of mGluR1 $\alpha$ is mediated by caveolin. J Neurochem 111:61-71.

Jeon D, Yang YM, Jeong MJ, Philipson KD, Rhim H, Shin HS (2003) Enhanced learning and memory in mice lacking $\mathrm{Na}^{+} / \mathrm{Ca}^{2+}$ exchanger 2 . Neuron 38:965-976.

Kaech S, Banker G (2006) Culturing hippocampal neurons. Nat Protoc 1:2406-2415.

Kakinuma N, Kiyama R (2009) A major mutation of KIF21A associated 
with congenital fibrosis of the extraocular muscles type 1 (CFEOM1) enhances translocation of Kank1 to the membrane. Biochem Biophys Res Commun 386:639-644.

Kiedrowski L (2004) High activity of $\mathrm{K}^{+}$-dependent plasmalemmal $\mathrm{Na}^{+}$/ $\mathrm{Ca}^{2+}$ exchangers in hippocampal CA1 neurons. Neuroreport 15: 2113-2116.

Kim MH, Lee SH, Park KH, Ho WK, Lee SH (2003) Distribution of $\mathrm{K}^{+}$dependent $\mathrm{Na}^{+} / \mathrm{Ca}^{2+}$ exchangers in the rat supraoptic magnocellular neuron is polarized to axon terminals. J Neurosci 23:11673-11680.

Kim MH, Korogod N, Schneggenburger R, Ho WK, Lee SH (2005) Interplay between $\mathrm{Na}^{+} / \mathrm{Ca}^{2+}$ exchangers and mitochondria in $\mathrm{Ca}^{2+}$ clearance at the calyx of Held. J Neurosci 25:6057-6065.

Kinjo TG, Szerencsei RT, Winkfein RJ, Kang K, Schnetkamp PP (2003) Topology of the retinal cone NCKX2 Na/Ca-K exchanger. Biochemistry 42:2485-2491.

Lee D, Lee KH, Ho WK, Lee SH (2007) Target cell-specific involvement of presynaptic mitochondria in post-tetanic potentiation at hippocampal mossy fiber synapses. J Neurosci 27:13603-13613.

Lee JS, Kim MH, Ho WK, Lee SH (2008) Presynaptic release probability and readily releasable pool size are regulated by two independent mechanisms during posttetanic potentiation at the calyx of Held synapse. J Neurosci 28:7945-7953.

Lee JY, Visser F, Lee JS, Lee KH, Soh JW, Ho WK, Lytton J, Lee SH (2006) Protein kinase C-dependent enhancement of activity of rat brain NCKX2 heterologously expressed in HEK293 cells. J Biol Chem 281:39205-39216.

Lee SH, Kim MH, Park KH, Earm YE, Ho WK (2002) $\mathrm{K}^{+}$-dependent $\mathrm{Na}^{+} /$ $\mathrm{Ca}^{2+}$ exchange is a major $\mathrm{Ca}^{2+}$ clearance mechanism in axon terminals of rat neurohypophysis. J Neurosci 22:6891-6899.

Lee SH, Park KH, Ho WK, Lee SH (2007) Postnatal developmental changes in $\mathrm{Ca}^{2+}$ homeostasis in supraoptic magnocellular neurons. Cell Calcium 41:441-450.

Lee SH, Ho WK, Lee SH (2009) Characterization of somatic $\mathrm{Ca}^{2+}$ clearance mechanisms in young and mature hippocampal granule cells. Cell Calcium 45:465-473.

Li Q, Lau A, Morris TJ, Guo L, Fordyce CB, Stanley EF (2004) A syntaxin 1, $\mathrm{G} \alpha_{\mathrm{o}}$, and N-type calcium channel complex at a presynaptic nerve terminal: analysis by quantitative immunocolocalization. J Neurosci 24: 4070-4081.

Li XF, Kiedrowski L, Tremblay F, Fernandez FR, Perizzolo M, Winkfein RJ, Turner RW, Bains JS, Rancourt DE, Lytton J (2006) Importance of $\mathrm{K}^{+}$dependent $\mathrm{Na}^{+} / \mathrm{Ca}^{2+}$-exchanger 2 , NCKX2, in motor learning and memory. J Biol Chem 281:6273-6282.

Lytton $\mathrm{J}$ (2007) $\mathrm{Na}^{+} / \mathrm{Ca}^{2+}$ exchangers: three mammalian gene families control $\mathrm{Ca}^{2+}$ transport. Biochem J 406:365-382.

Marszalek JR, Weiner JA, Farlow SJ, Chun J, Goldstein LS (1999) Novel dendritic kinesin sorting identified by different process targeting of two related kinesins: KIF21A and KIF21B. J Cell Biol 145:469-479.

Meijering E, Jacob M, Sarria JC, Steiner P, Hirling H, Unser M (2004) De- sign and validation of a tool for neurite tracing and analysis in fluorescence microscopy images. Cytometry A 58:167-176.

Oh YS, Cho KA, Ryu SJ, Khil LY, Jun HS, Yoon JW, Park SC (2006) Regulation of insulin response in skeletal muscle cell by caveolin status. J Cell Biochem 99:747-758.

Ohno H, Stewart J, Fournier MC, Bosshart H, Rhee I, Miyatake S, Saito T, Gallusser A, Kirchhausen T, Bonifacino JS (1995) Interaction of tyrosine-based sorting signals with clathrin-associated proteins. Science 269:1872-1875.

Rapoport I, Chen YC, Cupers P, Shoelson SE, Kirchhausen T (1998) Dileucine-based sorting signals bind to the beta chain of AP-1 at a site distinct and regulated differently from the tyrosine-based motif-binding site. EMBO J 17:2148-2155.

Regehr WG, Delaney KR, Tank DW (1994) The role of presynaptic calcium in short-term enhancement at the hippocampal mossy fiber synapse. J Neurosci 14:523-537.

Rubinson DA, Dillon CP, Kwiatkowski AV, Sievers C, Yang L, Kopinja J, Rooney DL, Zhang M, Ihrig MM, McManus MT, Gertler FB, Scott ML, Van Parijs L (2003) A lentivirus-based system to functionally silence genes in primary mammalian cells, stem cells and transgenic mice by RNA interference. Nat Genet 33:401-406.

Ryan XP, Alldritt J, Svenningsson P, Allen PB, Wu GY, Nairn AC, Greengard $P$ (2005) The Rho-specific GEF Lfc interacts with neurabin and spinophilin to regulate dendritic spine morphology. Neuron 47:85-100.

Sampo B, Kaech S, Kunz S, Banker G (2003) Two distinct mechanisms target membrane proteins to the axonal surface. Neuron 37:611-624.

Silverman MA, Kaech S, Ramser EM, Lu X, Lasarev MR, Nagalla S, Banker G (2010) Expression of kinesin superfamily genes in cultured hippocampal neurons. Cytoskeleton 67:784-795.

Song AH, Wang D, Chen G, Li Y, Luo J, Duan S, Poo MM (2009) A selective filter for cytoplasmic transport at the axon initial segment. Cell 136: $1148-1160$.

Tsoi M, Rhee KH, Bungard D, Li XF, Lee SL, Auer RN, Lytton J (1998) Molecular cloning of a novel potassium-dependent sodium-calcium exchanger from rat brain. J Biol Chem 273:4155-4162.

Visser F, Valsecchi V, Annunziato L, Lytton J (2007) Analysis of ion interactions with the $\mathrm{K}^{+}$-dependent $\mathrm{Na}^{+} / \mathrm{Ca}^{2+}$ exchangers NCKX2, NCKX3, and NCKX4: identification of Thr-551 as a key residue in defining the apparent $\mathrm{K}^{+}$affinity of NCKX2. J Biol Chem 282:4453-4462.

Xu M, Gu Y, Barry J, Gu C (2010) Kinesin I transports tetramerized Kv3 channels through the axon initial segment via direct binding. J Neurosci 30:15987-16001.

Yamada K, Andrews C, Chan WM, McKeown CA, Magli A, de Berardinis T, Loewenstein A, Lazar M, O'Keefe M, Letson R, London A, Ruttum M, Matsumoto N, Saito N, Morris L, Del Monte M, Johnson RH, Uyama E, Houtman WA, de Vries B, et al. (2003) Heterozygous mutations of the kinesin KIF21A in congenital fibrosis of the extraocular muscles type 1 (CFEOM1). Nat Genet 35:318-321. 